\title{
Formulas for the Reidemeister, Lefschetz and Nielsen coincidence number of maps between infra-nilmanifolds
}

\author{
Ku Yong $\mathrm{Ha}^{1}$, Jong Bum Lee ${ }^{1 *}$ and Pieter Penninckx ${ }^{2}$
}

\footnotetext{
* Correspondence: jlee@sogang.ac. $\mathrm{kr}$

'Department of Mathematics, Sogang University, Seoul 121-742, Korea

Full list of author information is available at the end of the article
}

\begin{abstract}
We prove practical formulas for the Reidemeister coincidence number, the Lefschetz coincidence number and the Nielsen coincidence number of continuous maps between oriented infra-nilmanifolds of equal dimension. In order to obtain these formulas, we use the averaging formulas for the Lefschetz coincidence number and for the Nielsen coincidence number and we develop an averaging formula for the Reidemeister coincidence number. We also give a simple proof of the averaging formula for the Lefschetz coincidence number.

Mathematics Subject Classification 2000: 55M20; 57530.
\end{abstract}

Keywords: infra-nilmanifold, Lefschetz coincidence number, Nielsen coincidence number, Reidemeister coincidence number.

\section{Introduction}

In order to study the number of fixed points of a continuous selfmap $f: M \rightarrow M$ on a closed, connected manifold $M$, three homotopy invariant numbers are associated to $f$ : the Reidemeister number $R(f)$, the Lefschetz number $L(f)$ and the Nielsen number $N(f)$. The non-vanishing of the Lefschetz number of $f$ implies the existence of a fixed point, while the Nielsen number is a lower bound for the number of fixed points. The Nielsen number is of particular interest since a classical result by Wecken [1] states that the Nielsen number coincides with the minimal number of fixed point in the homotopy class of the map when the dimension of $M$ is at least three.

Because of the mixture of geometry and algebra that occur in the definition of the Nielsen number, its computation is very hard in general and it is up to now the subject of a great deal of research. Simple and practical formulas have only been obtained in specific cases (for an overview, see for instance [2,3]) and one often turns the attention to comparing the Nielsen number to other numbers that are relatively more easy to compute, such as the Lefschetz number and the Reidemeister number (see for instance [4] for an overview).

Closely related to fixed point theory is coincidence theory. A point $x \in M_{1}$ is a coincidence of a pair of continuous maps $f, g: M_{1} \rightarrow M_{2}$ when $f(x)=g(x)$. In the case where $M_{1}$ and $M_{2}$ are both oriented and of equal dimension, the Reidemeister coincidence number $R(f, g)$, the Lefschetz coincidence number $L(f, g)$ and the Nielsen coincidence number $N(f, g)$ are defined. The Nielsen coincidence number is particularly

(C) $2012 \mathrm{Ha}$ et al; licensee Springer. This is an Open Access article distributed under the terms of the Creative Commons Attribution License (http://creativecommons.org/licenses/by/2.0), which permits unrestricted use, distribution, and reproduction in any medium, provided the original work is properly cited. 
interesting since it is, just as in the fixed point case, a strong lower bound for the number of coincidences. Unfortunately, the Nielsen coincidence number is usually at least as hard to compute as the Nielsen number in fixed point theory.

In 1985, Anosov [5] shows that for nilmanifolds, the Nielsen number can easily be computed via the Lefschetz number since $N(f)=|L(f)|$ for any continuous selfmap $f$ on a nilmanifold. The result of Anosov was also proved by Fadell and Husseini [6]. This is the beginning of the fruitful study of fixed point theory and coincidence theory for larger classes of manifolds, such as infra-nilmanifolds [7,8], solvmanifolds (see for instance [9]) and infra-solvmanifolds (see for example [10]). In [11,12], formulas for the Lefschetz (fixed point) number and the Nielsen (fixed point) number of continuous selfmaps on infra-nilmanifolds have been proved. In coincidence theory however, only recently a formula has been proved for the Lefschetz coincidence number and the Nielsen coincidence number of a pair of continuous maps between solvmanifolds of type $(R)$ [13]. For infra-nilmanifolds however, formulas for the Lefschetz coincidence number and the Nielsen coincidence number are still open for study.

In this article, we address this problem and we prove in Theorem 6.11 explicit and practical formulas for the Reidemeister coincidence number, the Lefschetz coincidence number and the Nielsen coincidence number of a pair of continuous maps between oriented infra-nilmanifolds of equal dimension, generalizing [12] from fixed point theory to coincidence theory and generalizing [13] from nilmanifolds to infra-nilmanifolds. In order to prove these formulas, we use the averaging formulas for the Nielsen coincidence number [14] and for the Lefschetz coincidence number (see [[9], p. 88] and [10]) and we develop an averaging formula for the Reidemeister coincidence number. We also formulate a simple proof for the averaging formula for the Lefschetz coincidence number.

\section{Preliminaries}

In this section, we fix notation and we give some definitions that will be needed to prove our results.

Definition 2.1. If $\widetilde{M} \rightarrow M$ is a covering map, then we use $A(\widetilde{M}, p)$ or simply $A(\tilde{M})$ to denote the covering transformation group. If $p_{1}: \widetilde{M}_{1} \rightarrow M_{1}$ and $p_{2}: \widetilde{M}_{2} \rightarrow M_{2}$ are covering maps, then we say that a continuous map $\tilde{f}: \widetilde{M}_{1} \rightarrow \widetilde{M}_{2}$ is a homotopy lift of a continuous map $f: M_{1} \rightarrow M_{2}$ when $\tilde{f}$ is the lift of a map homotopic to $f$.

Definition 2.2. Let $G$ be a group and $g \in G$. Then we use $\tau_{g}: G \rightarrow G: g^{\prime} \mapsto g g^{\prime} g^{-1}$ to denote the conjugation map $\lambda_{g}: G \rightarrow G: g^{\prime} \mapsto g g^{\prime}$ to denote the left multiplication map. If $\phi, \psi: G \rightarrow H$ are morphisms of groups, then we define $\operatorname{coin}(\phi, \psi)=\{g \in G \mid$ $\phi(g)=\psi(g)\}$.

\subsection{Coincidence theory}

In this section, we introduce basic notions concerning coincidence theory. A reference on coincidence theory is [15].

Let $M_{1}$ and $M_{2}$ be oriented, closed, connected manifolds of equal dimension. In order to study the coincidence set $\operatorname{Coin}(f, g)=\left\{x \in M_{1} \mid f(x)=g(x)\right\}$ of a pair of continuous maps $f, g: M_{1} \rightarrow M_{2}$, one splits the coincidence set into so-called coincidence classes. In this article, we define coincidence classes by fixing lifts $\tilde{f}, \tilde{g}: \widetilde{M}_{1} \rightarrow \widetilde{M}_{2}$ of $f$ 
and $g$, where $p_{1}: \widetilde{M}_{1} \rightarrow M_{1}$ and $p_{2}: \widetilde{M}_{2} \rightarrow M_{2}$ are universal covers. Remark that by fixing a lift $\tilde{f}: \widetilde{M}_{1} \rightarrow \widetilde{M}_{2}$ of $f: M_{1} \rightarrow M_{2}$, the continuous map $f: M_{1} \rightarrow M_{2}$ induces a morphism $f_{\times}: A\left(\widetilde{M}_{1}, p_{1}\right) \rightarrow A\left(\widetilde{M}_{2}, p_{2}\right)$ between the covering transformation groups as follows.

Definition 2.3. Let $A\left(\widetilde{M_{1}}, p_{1}\right)$ be the covering transformation group of the cover $p_{1}: \widetilde{M}_{1} \rightarrow M_{1}$ and $A\left(\widetilde{M}_{2}, p_{2}\right)$ the covering transformation group of the cover $p_{2}: \widetilde{M}_{2} \rightarrow M_{2}$. For every $\alpha \in A\left(\widetilde{M}_{1}, p_{1}\right)$, let $f_{\times}(\alpha)$ be the unique covering transformation in $A\left(\widetilde{M_{2}}, p_{2}\right)$ that satisfies $\tilde{f} \alpha=f_{\times}(\alpha) \tilde{f}$.

Fix a lift $\tilde{f}: \widetilde{M}_{1} \rightarrow \widetilde{M}_{2}$ of $f$ and a lift $\tilde{g}: \widetilde{M}_{1} \rightarrow \widetilde{M}_{2}$ of $g$. Then for any $\alpha \in A\left(\widetilde{M}_{2}, p_{2}\right)$, the set $p_{1}(\operatorname{Coin}(\alpha \tilde{f}, \tilde{g}))$ is by definition a coincidence class of $f, g$. Now for any pair of covering transformations $\alpha, \beta \in A\left(\widetilde{M}_{2}, p_{2}\right)$, if $p_{1}(\operatorname{Coin}(\alpha \tilde{f}, \tilde{g})) \cap p_{1}(\operatorname{Coin}(\beta \tilde{f}, \tilde{g})) \neq \emptyset$, then one can show that $p_{1}(\operatorname{Coin}(\alpha \tilde{f}, \tilde{g}))=p_{1}(\operatorname{Coin}(\beta \tilde{f}, \tilde{g}))$ and that there exists $\gamma \in A\left(\tilde{M}_{1}, p_{1}\right)$ such that $\beta=g_{\times}(\gamma) \alpha f_{\times}(\gamma)^{-1}$. This is the motivation for the following definitions.

Definition 2.4. Let $G_{1}$ and $G_{2}$ be groups and $\phi, \psi: G_{1} \rightarrow G_{2}$ morphisms of groups. Define an equivalence relation $\sim$ on $G_{2}$ by

$$
\alpha \sim \beta \Leftrightarrow \exists \gamma \in G_{1}: \beta=\psi(\gamma) \alpha \varphi(\gamma)^{-1} .
$$

The equivalence classes are called coincidence Reidemeister classes or (doubly) twisted conjugacy classes and $\mathcal{R}[\varphi, \psi]$ denotes the set of coincidence Reidemeister classes. For any $\alpha \in G_{2}$, we use $[\alpha]$ to denote the coincidence Reidemeister class containing $\alpha$. The Reidemeister coincidence number $R(\phi, \psi)$ of $\phi, \psi$ is defined as the cardinality of $\mathcal{R}[\varphi, \psi]$.

Definition 2.5. The Reidemeister coincidence number $R(f, g)$ of the continuous maps $f$ and $g$ is defined as the Reidemeister coincidence number $R\left(f_{x}, g_{x}\right)$ of the induced morphisms $f_{\times}$and $g_{\times}$. For any coincidence Reidemeister class $[\alpha] \in \mathcal{R}\left[f_{\times}, g_{\times}\right]$, the set $p_{1}($ Coin $(\alpha \tilde{f}, \tilde{g}))$ does not depend on the particular choice of the representative $\alpha$ of the coincidence Reidemeister class and we call $p_{1}(\operatorname{Coin}(\alpha \tilde{f}, \tilde{g}))$ the coincidence class of $f, g$ corresponding to $[\alpha]$.

Note that the Reidemeister coincidence number $R(f, g)$ does not depend on the particular choice of the lifts $\tilde{f}, \tilde{g}$. Also the coincidence classes do not depend on the choice of lifts, although the corresponding coincidence Reidemeister classes may.

To each isolated subset $C$ of $\operatorname{Coin}(f, g)$, one associates an integer ind $(f, g ; C)$, called the coincidence index, which generalizes the well-known fixed point index to Nielsen coincidence theory in the setting of maps between oriented manifolds of the equal dimension by Schirmer [16] (see also [17]). Each coincidence class is an isolated subset of Coin $(f, g)$. If the coincidence index of a coincidence class is non-zero, then we call the coincidence class essential. One can prove that the number of essential coincidence classes is finite. The Lefschetz coincidence number $L(f, g)$ is by definition the sum of the coincidence indices of the coincidence classes. If $L(f, g) \neq 0$, then $f$ and $g$ have a coincidence. The Nielsen coincidence number $N(f, g)$ is defined as the number of 
essential coincidence classes of $f, g$. This number plays a central role in coincidence theory since $N(f, g)$ is a lower bound for the cardinality of Coin $(f, g)$. The Nielsen coincidence number, the Lefschetz coincidence number and the Reidemeister coincidence numbers are homotopy invariants: if $f$ is homotopic to $f$ and $g$ ' is homotopic to $g$, then $N\left(f, g^{\prime}\right)=N(f, g), L\left(f^{\prime}, g^{\prime}\right)=L(f, g)$ and $R\left(f, g^{\prime}\right)=R(f, g)$. Schirmer [16] shows that when the dimension of $M_{1}$ and $M_{2}$ is at least three, then for any pair of continuous maps $f$, $g: M_{1} \rightarrow M_{2}$, there exist maps $f^{\prime}$ homotopic to $f$ and $g^{\prime}$ homotopic to $g$ such that the cardinality of $\operatorname{Coin}\left(f, g^{\prime}\right)$ is precisely $N(f, g)$. In other words: when the dimension is at least three, then the Nielsen number coincides with the minimal number of coincidence points in the homotopy class of the map.

\subsection{Infra-nilmanifolds}

In this section, we shortly review infra-nilmanifolds. A reference is [18]. Let $G$ be a connected, simply connected, nilpotent Lie group and let $C$ be a maximal compact subgroup of $\operatorname{Aut}(G)$. A discrete and cocompact subgroup $\Pi$ of $G \rtimes C \subset \operatorname{Aff}(G)=G \rtimes$ $\operatorname{Aut}(G)$ is called an almost-crystallographic group. Moreover, if $\Pi$ is torsion free, then $\Pi$ is called an almost-Bieberbach group and the quotient space $\Pi \backslash G$ is a closed manifold that we call an infra-nilmanifold. In particular, if $\Pi \subset G$, then $\Pi \backslash G$ is called a nilmanifold. Recall from [19] that $\Gamma=\Pi \cap G$ is the maximal normal nilpotent subgroup of $\Pi$ with finite quotient group $\Pi / \Gamma$. The quotient $\Pi / \Gamma$ is isomorphic to the group $\{A$ $\in \operatorname{Aut}(G) \mid \exists a \in G$ such that $(a, A) \in \Pi\}$ which we call the holonomy group of the infra-nilmanifold $\Pi \backslash G$ or of the almost-Bieberbach group $\Pi$.

Let us recall an important result on maps between infra-nilmanifolds.

Theorem 2.6. [[8], Corollary 1.2] Let $G_{1}$ and $G_{2}$ be connected, simply connected, nilpotent Lie groups and $M_{1}$ and $M_{2}$ infra-nilmanifolds modeled on $G_{1}$ and $G_{2}$ respectively. Let $f: M_{1} \rightarrow M_{2}$ be a continuous map, then there exist $d \in G_{2}$ and a morphism of Lie groups $D: G_{1} \rightarrow G_{2}$ such that $\lambda_{d} \circ D: G_{1} \rightarrow G_{2}$ is a homotopy lift of $f$.

In fact, the result in [8] is only formulated for selfmaps, but it is straight forward to generalize the proof to the setting in Theorem 2.6. There is a similar result for maps between nilmanifolds, cf. [[6], Proposition 3.2] or [[9], Lemma 2.7]:

Theorem 2.7. Let $G_{1}$ and $G_{2}$ be connected, simply connected, nilpotent Lie groups and $N_{1}$ and $N_{2}$ nilmanifolds modeled on $G_{1}$ and $G_{2}$ respectively. Let $f: N_{1} \rightarrow N_{2}$ be a continuous map. Then $f$ has a homotopy lift $D: G_{1} \rightarrow G_{2}$ that is a morphism of Lie groups.

When $D: G_{1} \rightarrow G_{2}$ is a morphism of Lie groups, then we will use $D$ * to denote the corresponding morphism of Lie algebras.

\section{The Reidemeister coincidence number}

Suppose we have a commutative diagram of groups:

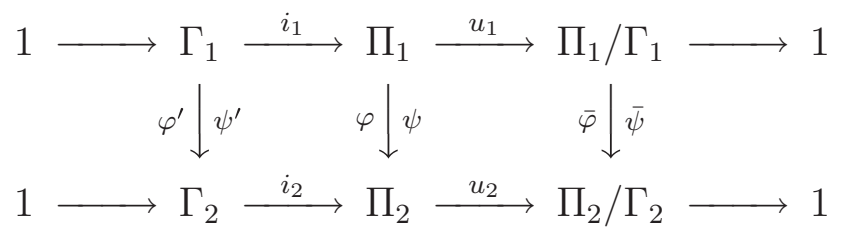


where the top and bottom sequences are exact and where the quotient groups $\Pi_{1} / \Gamma_{1}$ and $\Pi_{2} / \Gamma_{2}$ are finite. For each $\bar{\alpha} \in \Pi_{2} / \Gamma_{2}$ and $\alpha \in u_{2}^{-1}(\bar{\alpha})$, we have a commutative diagram

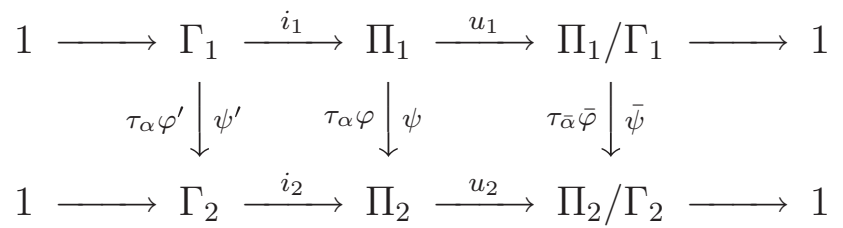

Moreover the following sequence of groups

$$
1 \rightarrow \operatorname{coin}\left(\tau_{\alpha} \varphi^{\prime}, \psi^{\prime}\right) \stackrel{i_{1}}{\rightarrow} \operatorname{coin}\left(\tau_{\alpha} \varphi, \psi\right) \stackrel{u_{1}}{\rightarrow} \operatorname{coin}\left(\tau_{\bar{\alpha}} \bar{\varphi}, \bar{\psi}\right)
$$

is exact. Remark that $i_{2}: \Gamma_{2} \rightarrow \Pi_{2}$ and $u_{2}: \Pi_{2} \rightarrow \Pi_{2} / \Gamma_{2}$ induce maps $\hat{i}_{2}^{\alpha}: \mathcal{R}\left[\tau_{\alpha} \varphi^{\prime}, \psi^{\prime}\right] \rightarrow \mathcal{R}\left[\tau_{\alpha} \varphi, \psi\right]$ and $\hat{u}_{2}^{\alpha}: \mathcal{R}\left[\tau_{\alpha} \varphi, \psi\right] \rightarrow \mathcal{R}\left[\tau_{\bar{\alpha}} \bar{\varphi}, \bar{\psi}\right]$ such that $\hat{u}_{2}^{\alpha}$ is surjective and $\left(\hat{u}_{2}^{\alpha}\right)^{-1}([\overline{1}])=\operatorname{im}\left(\hat{i}_{2}^{\alpha}\right)$. Define $\hat{i}_{2}=\hat{i}_{2}^{\mathrm{Id}}$ and $\hat{u}_{2}=\hat{u}_{2}^{\mathrm{Id}}$, where Id $\in \Pi_{2}$ is the identity element.

With this notation:

Lemma 3.1. [[14], Lemma 2.1] Given the commutative diagram (3.1), we have for each $\alpha \in \Pi_{2}$ that

$$
\begin{aligned}
& \begin{array}{l}
\# \hat{u}_{2}^{-1}([\bar{\alpha}])=\#\left(\hat{u}_{2}^{\alpha}\right)^{-1}([\overline{1}]), \\
\begin{aligned}
R(\varphi, \psi) & =\sum_{[\bar{\alpha}] \in \mathcal{R}[\bar{\varphi}, \bar{\psi}]} \# \hat{u}_{2}^{-1}([\bar{\alpha}])=\sum_{[\bar{\alpha}] \in \mathcal{R}[\bar{\varphi}, \bar{\psi}]} \#\left(\hat{u}_{2}^{\alpha}\right)^{-1}([\overline{1}]) \\
& =\sum_{[\bar{\alpha}] \in \mathcal{R}[\bar{\varphi}, \bar{\psi}]} \# \operatorname{im}\left(\hat{i}_{2}^{\alpha}\right),
\end{aligned} \\
R\left(\tau_{\alpha} \varphi^{\prime}, \psi^{\prime}\right)=\sum_{[\gamma] \in \operatorname{im}\left(\hat{i}_{2}^{\alpha}\right)} \#\left(\hat{i}_{2}^{\alpha}\right)^{-1}([\gamma]), \\
\#\left(\hat{i}_{2}^{\alpha}\right)^{-1}([\gamma])=\left[\operatorname{coin}\left(\tau_{\bar{\alpha}} \bar{\varphi}, \bar{\psi}\right): u_{1}\left(\operatorname{coin}\left(\tau_{\gamma \alpha} \varphi, \psi\right)\right)\right] \text { for each } \gamma \in \Gamma_{2}, \\
R\left(\tau_{\alpha} \varphi^{\prime}, \psi^{\prime}\right)=\sum_{[\gamma] \in \operatorname{im}\left(\hat{i}_{2}^{\alpha}\right)}\left[\operatorname{coin}\left(\tau_{\bar{\alpha}} \bar{\varphi}, \bar{\psi}\right): u_{1}\left(\operatorname{coin}\left(\tau_{\gamma \alpha} \varphi, \psi\right)\right)\right] .
\end{array}
\end{aligned}
$$

This lemma is stated in [[14], Lemma 2.1] as a straightforward extension of [[11], Lemma 2.1] in which a topological proof of (4) is given. We will translate the topological proof to algebra.

Proof. Choose arbitrary $\alpha \in \Pi_{2}$.

(1) Choose arbitrary $\gamma \in \Pi_{2}$ and define $\bar{\gamma}=u_{2}(\gamma)$. Then

$$
\begin{aligned}
& {[\gamma] \in \hat{u}_{2}^{-1}([\bar{\alpha}]) \Leftrightarrow[\bar{\gamma}]=[\bar{\alpha}] \text { in } \mathcal{R}[\bar{\varphi}, \bar{\psi}] } \\
& \Leftrightarrow \exists \bar{\delta} \in \Pi_{1} / \Gamma_{1} \text { such that } \bar{\gamma} \bar{\alpha}^{-1}=\bar{\psi}(\bar{\delta}) \bar{\alpha} \bar{\varphi}(\bar{\delta})^{-1} \bar{\alpha}^{-1} \\
& \qquad=\bar{\varphi}(\bar{\delta}) \overline{1}\left(\tau_{\bar{\alpha}} \bar{\varphi}\right)(\bar{\delta})^{-1} \\
& \Leftrightarrow \hat{u}_{2}^{\alpha}\left(\left[\gamma \alpha^{-1}\right]\right)=\left[\bar{\gamma} \bar{\alpha}^{-1}\right]=[\overline{1}] \text { in } \mathcal{R}\left[\tau_{\bar{\alpha}} \bar{\varphi}, \bar{\psi}\right] \\
& \Leftrightarrow\left[\gamma \alpha^{-1}\right] \in\left(\hat{u}_{2}^{\alpha}\right)^{-1}([\overline{1}]) .
\end{aligned}
$$


(2) Because $\hat{u}_{2}$ is surjective, $\mathcal{R}[\varphi, \psi]$ equals the disjoint union

$$
\mathcal{R}[\varphi, \psi]=\coprod_{[\bar{\alpha}] \in \mathcal{R}[\bar{\varphi}, \bar{\psi}]} \hat{u}_{2}^{-1}([\bar{\alpha}]) .
$$

Hence the first equality follows. The second equality follows from (1) and the last equality follows from the fact that $\left(\hat{u}_{2}^{\alpha}\right)^{-1}([\overline{1}])=\operatorname{im}\left(\hat{i}_{2}^{\alpha}\right)$.

(3) This follows from the fact that $\mathcal{R}\left[\tau_{\alpha} \varphi^{\prime}, \psi^{\prime}\right]$ equals the disjoint union

$$
\mathcal{R}\left[\tau_{\alpha} \varphi^{\prime}, \psi^{\prime}\right]=\coprod_{[\gamma] \in \operatorname{im}\left(\hat{i}_{3}^{\alpha}\right)}\left(\hat{i}_{2}^{\alpha}\right)^{-1}([\gamma]) .
$$

(4) Choose arbitrary $\gamma \in \Gamma_{2}$. Define

$$
A: \operatorname{coin}\left(\tau_{\bar{\alpha}} \bar{\varphi}, \bar{\psi}\right) \rightarrow\left(\hat{i}_{2}^{\alpha}\right)^{-1}([\gamma]): \bar{\delta} \mapsto A(\bar{\delta}),
$$

where

$$
A(\bar{\delta})=\left[\psi(\delta) \gamma\left(\tau_{\alpha} \varphi\right)(\delta)^{-1}\right] \in\left(\hat{i}_{2}^{\alpha}\right)^{-1}([\gamma]) \subset \mathcal{R}\left[\tau_{\alpha} \varphi^{\prime}, \psi^{\prime}\right],
$$

and where $\delta \in \Pi_{1}$ is chosen such that $u_{1}(\delta)=\bar{\delta}$.

First we prove that $A$ is well defined:

$\psi(\delta) \gamma\left(\tau_{\alpha} \phi\right)(\delta)^{-1}$ belongs to $\Gamma_{2}$ : This follows from the fact that $u_{2}\left(\psi(\delta) \gamma\left(\tau_{\alpha} \varphi\right)(\delta)^{-1}\right)=\bar{\psi}(\bar{\delta})\left(\tau_{\bar{\alpha}} \bar{\varphi}\right)(\bar{\delta})^{-1}=1$ because $\bar{\delta} \in \operatorname{coin}\left(\tau_{\bar{\alpha}} \bar{\varphi}, \bar{\psi}\right)$.

$\left[\psi(\delta) \gamma\left(\tau_{\alpha} \phi\right)(\delta)^{-1}\right]$ does not depend on the choice of $\delta$ :

Suppose that $u_{1}(\delta)=u_{1}(\delta \prime)=\bar{\delta}$. Then there exists $\beta \in \Gamma_{1}$ such that $\delta^{\prime}=\beta \delta$.

Then

$$
\psi\left(\delta^{\prime}\right) \gamma\left(\tau_{\alpha} \varphi\right)\left(\delta^{\prime}\right)^{-1}=\psi^{\prime}(\beta)\left(\psi(\delta) \gamma\left(\tau_{\alpha} \varphi\right)(\delta)^{-1}\right)\left(\tau_{\alpha} \varphi^{\prime}\right)(\beta)^{-1}
$$

so that

$$
\left[\psi\left(\delta^{\prime}\right) \gamma\left(\tau_{\alpha} \varphi\right)\left(\delta^{\prime}\right)^{-1}\right]=\left[\psi(\delta) \gamma\left(\tau_{\alpha} \varphi\right)(\delta)^{-1}\right] .
$$

Let us now prove that $A$ is surjective. Choose arbitrary $[\beta] \in\left(\hat{i}_{2}^{\alpha}\right)^{-1}([\gamma])$. Because $\left(\hat{i}_{2}^{\alpha}\right)([\beta])=[\gamma]$, there exists $\delta \in \Pi_{1}$ such that $\beta=\psi(\delta) \gamma\left(\tau_{\alpha} \phi\right)(\delta)^{-1}$. Because $\beta, \gamma \in \Gamma_{2}$, we have that $1=u_{2}(\beta)=\bar{\psi}(\bar{\delta})\left(\tau_{\bar{\alpha}} \bar{\varphi}\right)(\bar{\delta})^{-1}$, where $\bar{\delta}=u_{2}(\delta)$ so that $\bar{\delta} \in \operatorname{coin}\left(\tau_{\bar{\alpha}} \bar{\varphi}, \bar{\psi}\right)$. Additionally, $A(\bar{\delta})=[\beta]$.

In order to prove statement (4), it is left to prove that for each $\bar{\delta}, \bar{\delta}^{\prime} \in \operatorname{coin}\left(\tau_{\bar{\alpha}} \bar{\varphi}, \bar{\psi}\right)$, we have that $A(\bar{\delta})=A(\bar{\delta} \prime)$ if and only if there exists $\beta \in \operatorname{coin}\left(\tau_{\gamma \alpha} \phi, \psi\right)$ such that $\bar{\delta}^{\prime}=\bar{\delta} u_{1}(\beta)$. Choose arbitrary $\bar{\delta}, \bar{\delta}^{\prime} \in \operatorname{coin}\left(\tau_{\bar{\alpha}} \bar{\varphi}, \bar{\psi}\right)$.

First assume that there exists $\beta \in \operatorname{coin}\left(\tau_{\gamma \alpha} \phi, \psi\right)$ such that $\bar{\delta}^{\prime}=\bar{\delta} u_{1}(\beta)$. Choose $\delta \in \Pi_{1}$ such that $u_{1}(\delta)=\bar{\delta}$ and define $\delta^{\prime}=\delta \beta$, then $u_{1}\left(\delta^{\prime}\right)=\bar{\delta}^{\prime}$. Remark that because $\beta \in$ coin $\left(\tau_{\gamma \alpha} \phi, \psi\right)$ we have that $\psi(\beta)=\gamma\left(\tau_{\alpha} \phi\right)(\beta) \gamma^{-1}$ so that $\psi(\beta) \gamma\left(\tau_{\alpha} \phi\right)(\beta)^{-1}=\gamma$. Hence 


$$
\begin{aligned}
A\left(\bar{\delta}^{\prime}\right) & =\left[\psi\left(\delta^{\prime}\right) \gamma\left(\tau_{\alpha} \varphi\right)\left(\delta^{\prime}\right)^{-1}\right]=\left[\psi(\delta \beta) \gamma\left(\tau_{\alpha} \varphi\right)(\delta \beta)^{-1}\right] \\
& =\left[\psi(\delta) \psi(\beta) \gamma\left(\tau_{\alpha} \varphi\right)(\beta)^{-1}\left(\tau_{\alpha} \varphi\right)(\delta)^{-1}\right] \\
& =\left[\psi(\delta) \gamma\left(\tau_{\alpha} \varphi\right)(\delta)^{-1}\right]=A(\bar{\delta}) .
\end{aligned}
$$

Conversely suppose that $A(\bar{\delta})=A(\bar{\delta} \prime)$. It then suffices to prove that $\bar{\delta}^{-1} \bar{\delta}^{\prime} \in u_{1}\left(\operatorname{coin}\left(\tau_{\gamma \alpha} \varphi, \psi\right)\right)$. Choose $\delta, \delta^{\prime} \in \Pi_{1}$ such that $u_{1}(\delta)=\bar{\delta}$ and $\bar{\delta}^{\prime}=\bar{\delta} u_{1}(\beta)$. Then because $A\left(\bar{\delta}^{\prime}\right)=A(\bar{\delta})$, there exists $\eta \in \Gamma_{1}$ such that

$$
\begin{aligned}
\psi\left(\delta^{\prime}\right) \gamma\left(\tau_{\alpha} \varphi\right)\left(\delta^{\prime}\right)^{-1} & =\psi(\eta) \psi(\delta) \gamma\left(\tau_{\alpha} \varphi\right)(\delta)^{-1}\left(\tau_{\alpha} \varphi\right)(\eta)^{-1}, \\
& =\psi(\eta \delta) \gamma\left(\tau_{\alpha} \varphi\right)(\eta \delta)^{-1}
\end{aligned}
$$

or

$$
\psi\left(\delta^{-1} \eta^{-1} \delta^{\prime}\right) \gamma=\gamma\left(\tau_{\alpha} \varphi\right)\left(\delta^{-1} \eta^{-1} \delta^{\prime}\right) .
$$

Hence $\delta^{-1} \eta^{-1} \delta \prime \in \operatorname{coin}\left(\tau_{\gamma \alpha} \varphi, \psi\right)$ and $\bar{\delta}^{-1} \bar{\delta}^{\prime}=u_{1}\left(\delta^{-1} \eta^{-1} \delta^{\prime}\right) \in\left(\operatorname{coin}\left(\tau_{\gamma \alpha} \varphi, \psi\right)\right)$.

(5) This follows from (3) and (4).

Corollary 3.2. [[20], Corollary 2.1] Given the commutative diagram (3.1), $\operatorname{coin}\left(\tau_{\bar{\alpha}}, \bar{\psi}\right)=\{\overline{1}\}$ for all $\alpha \in \Pi_{2}$ then

$$
R(\varphi, \psi)=\sum_{[\bar{\alpha}] \in \mathcal{R}[\bar{\varphi}, \bar{\psi}]} R\left(\tau_{\alpha} \varphi^{\prime}, \psi^{\prime}\right) .
$$

Proof. By (2) of Lemma 3.1,

$$
R(\varphi, \psi)=\sum_{[\bar{\alpha}] \in \mathcal{R}[\bar{\varphi}, \bar{\psi}]} \# \operatorname{im}\left(\hat{i}_{2}^{\alpha}\right)=\sum_{[\bar{\alpha}] \in \mathcal{R}[\bar{\varphi}, \bar{\psi}]} \# i_{2}^{\alpha}\left(\mathcal{R}\left[\tau_{\alpha} \varphi^{\prime}, \psi^{\prime}\right]\right) .
$$

By (4) of Lemma 3.1, $\hat{i}_{2}^{\alpha}$ is injective and hence the conclusion follows.

Remark 3.3. The group $\Pi_{1} / \Gamma_{1}$ acts on the set $[\bar{\alpha}] \in \mathcal{R}[\bar{\varphi}, \bar{\psi}]$ by the rule $\bar{\alpha} \mapsto \bar{\psi}(\bar{\beta}) \bar{\alpha} \bar{\varphi}(\bar{\beta})^{-1}$. This action is transitive. The isotropy subgroup is

$$
\left\{\bar{\beta} \mid \bar{\psi}(\bar{\beta}) \bar{\alpha} \bar{\varphi}(\bar{\beta})^{-1}=\bar{\alpha}\right\}=\operatorname{coin}\left(\tau_{\bar{\alpha}} \bar{\varphi}, \bar{\psi}\right) .
$$

Hence $\left[\Pi_{1}: \Gamma_{1}\right]=\#[\bar{\alpha}] \cdot \# \operatorname{coin}\left(\tau_{\bar{\alpha}} \bar{\varphi}, \bar{\psi}\right)$.

Corollary 3.4. [[21], Proposition 3.8] Suppose we are given the commutative diagram (3.1). Then $R(\phi, \psi)$ is finite if and only if $R\left(\tau_{\alpha} \phi^{\prime}, \psi^{\prime}\right)$ is finite for every $\alpha \in \Pi_{2}$.

Proof. Suppose that $R(\phi, \psi)$ is finite. Choose arbitrary $\alpha \in \Pi_{2}$. By (2) of Lemma 3.1, $\operatorname{im}\left(\hat{i}_{2}^{\alpha}\right)$ is finite. Because also $\left(\tau_{\bar{\alpha}} \bar{\varphi}, \bar{\psi}\right)$ is finite, by (5) of Lemma 3.1, $R\left(\tau_{\alpha} \phi^{\prime}, \psi^{\prime}\right)$ is finite. The converse is also easy to check.

Furthermore, the Reidemeister coincidence numbers $R(\phi, \psi)$ and $R\left(\tau_{\alpha} \phi^{\prime}, \psi^{\prime}\right)$ are directly related as follows:

Theorem 3.5. Suppose we have a commutative diagram of groups:

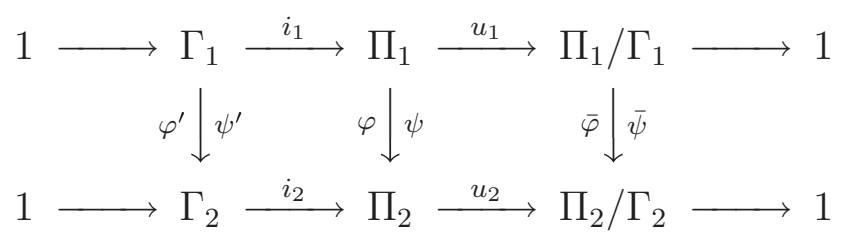


where the top and bottom sequences are exact and the quotient groups $\Pi_{1} / \Gamma_{1}$ and $\Pi_{2} /$ $\Gamma_{2}$ are finite. Then

$$
R(\varphi, \psi) \geq \frac{1}{\left[\Pi_{1}: \Gamma_{1}\right]} \sum_{\bar{\alpha} \in \Pi_{2} / \Gamma_{2}} R\left(\tau_{\alpha} \varphi^{\prime}, \psi^{\prime}\right) .
$$

When either side of the inequality is finite, then equality occurs if and only if coin $\left.\left(\tau_{\alpha} \phi, \psi\right)\right) \subset \Gamma_{1}$ for each $\alpha \in \Pi_{2}$.

Remark that $R\left(\tau_{\alpha} \phi^{\prime}, \psi^{\prime}\right)$ depends only on $\bar{\alpha}=u_{2}(\alpha)$. Indeed, suppose that $u_{2}(\alpha)=u_{2}$ $(\beta)$, then $\alpha \beta^{-1} \in \Gamma_{2}$ and $\beta \alpha^{-1} \in \Gamma_{2}$ and we can define

$$
A: \mathcal{R}\left[\tau_{\alpha} \varphi^{\prime}, \psi^{\prime}\right] \rightarrow \mathcal{R}\left[\tau_{\beta} \varphi^{\prime}, \psi^{\prime}\right]:[\gamma] \mapsto\left[\gamma \alpha \beta^{-1}\right]
$$

and

$$
B: \mathcal{R}\left[\tau_{\beta} \varphi^{\prime}, \psi^{\prime}\right] \rightarrow \mathcal{R}\left[\tau_{\alpha} \varphi^{\prime}, \psi^{\prime}\right]:[\gamma] \mapsto\left[\gamma \beta \alpha^{-1}\right] .
$$

$A$ and $B$ are bijections that are each other's inverse, hence $R\left(\tau_{\alpha} \phi^{\prime}, \psi^{\prime}\right)=R\left(\tau_{\beta} \phi^{\prime}, \psi^{\prime}\right)$.

Proof of Theorem 3.5. This follows from the following observations:

$$
\begin{aligned}
& \sum_{\bar{\alpha} \in \Pi_{2} / \Gamma_{2}} R\left(\tau_{\alpha} \varphi^{\prime}, \psi^{\prime}\right) \\
& =\sum_{\bar{\alpha} \in \Pi_{2} / \Gamma_{2}} \sum_{[\gamma] \in \operatorname{im}\left(\hat{i}_{2}^{\alpha}\right)}\left[\operatorname{coin}\left(\tau_{\bar{\alpha}} \bar{\varphi}, \bar{\psi}\right): u_{1}\left(\operatorname{coin}\left(\tau_{\gamma \alpha} \varphi, \psi\right)\right)\right] \\
& \text { by (5) of Lemma } 3.1 \\
& =\left[\Pi_{1}: \Gamma_{1}\right] \sum_{\bar{\alpha} \in \Pi_{2} / \Gamma_{2}} \sum_{[\gamma] \in \operatorname{im}\left(\hat{i}_{2}^{\alpha}\right)} \frac{\left[\operatorname{coin}\left(\tau_{\bar{\alpha}} \bar{\varphi}, \bar{\psi}\right): u_{1}\left(\operatorname{coin}\left(\tau_{\gamma \alpha} \varphi, \psi\right)\right)\right]}{\#[\bar{\alpha}] \cdot \# \operatorname{coin}\left(\tau_{\bar{\alpha}} \bar{\varphi}, \bar{\psi}\right)} \\
& =\left[\Pi_{1}: \Gamma_{1}\right] \sum_{\bar{\alpha} \in \Pi_{2} / \Gamma_{2}} \frac{1}{\#[\bar{\alpha}]} \sum_{[\gamma] \in i m\left(i_{2}^{\alpha}\right)} \frac{1}{\# u_{1}\left(\operatorname{coin}\left(\tau_{\gamma \alpha} \varphi, \psi\right)\right)} \\
& \leq\left[\Pi_{1}: \Gamma_{1}\right] \sum_{\bar{\alpha} \in \Pi_{2} / \Gamma_{2}} \frac{1}{\#[\bar{\alpha}]} \sum_{[\gamma] \in i m\left(\hat{i}_{2}^{\alpha}\right)} 1 \\
& =\left[\Pi_{1}: \Gamma_{1}\right] \sum_{\bar{\alpha} \in \mathcal{R}[\bar{\varphi}, \bar{\psi}]} \# \operatorname{im}\left(\hat{i}_{2}^{\alpha}\right) \\
& =\left[\Pi_{1}: \Gamma_{1}\right] \cdot R(\varphi, \psi) \\
& \text { by (2) of Lemma 3.1. }
\end{aligned}
$$

Moreover, when either side of the inequality is finite, then equality holds if and only if for each $\alpha \in \Pi_{2}, u_{1}\left(\operatorname{coin}\left(\tau_{\alpha} \phi, \psi\right)\right)$ is the trivial group.

\section{Reidemeister coincidence numbers for covering spaces}

Now we can translate the previous results on algebraic Reidemeister coincidence numbers to results on topological Reidemeister coincidence numbers.

Theorem 4.1. Let $M_{1}$ and $M_{2}$ be closed and connected manifolds with universal covers $p_{1}: \widetilde{M}_{1} \rightarrow M_{1}$ and $p_{2}: \widetilde{M}_{2} \rightarrow M_{2}$. Let $(f, g): M_{1} \rightarrow M_{2}$ be a pair of maps and let $(\tilde{f}, \tilde{g}): \widetilde{M}_{1} \rightarrow \widetilde{M}_{2}$ be a pair of lifts of $(f, g)$. Let $\Pi_{1}=A\left(\tilde{M}_{1}, p_{1}\right)$ and $\Pi_{2}=A\left(\widetilde{M}_{2}, p_{2}\right)$ be 
the covering transformation groups and let $f_{\times}, g_{\times}: \Pi_{1} \rightarrow \Pi_{2}$ be the morphisms of groups induced by $(\tilde{f}, \tilde{g})$. Suppose $\Gamma_{1}$ is a finite index normal subgroup of $\Pi_{1}$ and $\Gamma_{2}$ is a finite index normal subgroup of $\Pi_{2}$ such that $f_{\times}\left(\Gamma_{1}\right) \subset \Gamma_{2}$ and $g_{\times}\left(\Gamma_{1}\right) \subset \Gamma_{2}$. Let $(\bar{f}, \bar{g}): \Gamma_{1} \backslash \widetilde{M}_{1} \rightarrow \Gamma_{2} \backslash \widetilde{M}_{2}$ be a pair of lifts of $(f, g)$ so that the following diagram commutes

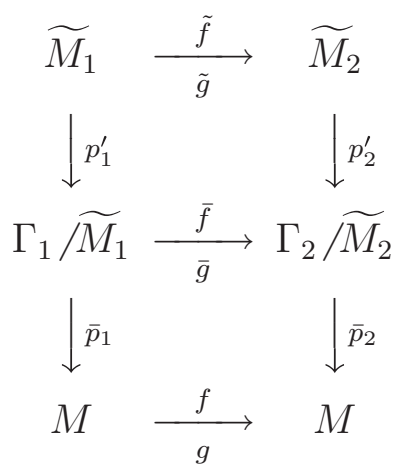

Then:

(1) If $\operatorname{coin}\left(\tau_{\bar{\alpha}} \bar{f}_{\times}, \bar{g}_{\times}\right)=\{\overline{1}\}$ for all $\alpha \in \Pi_{2}$, then

$$
R(f, g)=\sum_{[\bar{\alpha}] \in \mathcal{R}\left[\bar{f}_{x}, \bar{g}_{x}\right]} R(\bar{\alpha} \bar{f}, \bar{g}) .
$$

(2) $R(f, g)$ is finite if and only if $R(\bar{\alpha} \bar{f}, \bar{g})$ is finite for every $\alpha \in \Pi_{2}$.

(3) We have

$$
R(f, g) \geq \frac{1}{\left[\Pi_{1}: \Gamma_{1}\right]} \sum_{\bar{\alpha} \in A\left(\Gamma_{2} \backslash \widetilde{M}_{2}, \bar{p}_{2}\right)} R(\bar{\alpha} \bar{f}, \bar{g}) .
$$

When either side of the inequality is finite, then equality occurs if and only if coin $\left(\tau_{\alpha} f_{\times}, g_{\times}\right) \subset \Gamma_{1}$ for each $\alpha \in \Pi_{2}$.

Theorem 4.2 (Averaging Formula for the Reidemeister Coincidence Number). Let $M_{1}$ and $M_{2}$ be orientable infra-nilmanifolds of equal dimension modeled on connected, simply connected, nilpotent Lie groups $G_{1}$ and $G_{2}$ respectively. Let $(f, g): M_{1} \rightarrow M_{2}$ be a pair of maps. Let $\Pi_{1}=A\left(G_{1}, p_{1}\right)$ and $\Pi_{2}=A\left(G_{2}, p_{2}\right)$ be the covering transformation groups and let $f_{\times}, g_{\times}: \Pi_{1} \rightarrow \Pi_{2}$ be the morphisms of groups induced by $f$, $g$ respectively. Let $\Gamma_{1}$ and $\Gamma_{2}$ be finite index normal subgroups of $\Pi_{1}$ and $\Pi_{2}$ respectively such that $f_{\times}\left(\Gamma_{1}\right) \subset \Gamma_{2}$ and $g_{\times}\left(\Gamma_{1}\right) \subset \Gamma_{2}$ and such that $N_{1}=\Gamma_{1} \backslash G_{1}$ and $N_{2}=\Gamma_{2} \backslash G_{2}$ are nilmanifolds. If $(\bar{f}, \bar{g}): N_{1} \rightarrow N_{2}$ is a pair of lifts of $(f, g)$, then

$$
R(f, g)=\frac{1}{\left[\Pi_{1}: \Gamma_{1}\right]} \sum_{\bar{\alpha} \in A\left(N_{2}, \bar{p}_{2}\right)} R(\bar{\alpha} \bar{f}, \bar{g}) .
$$

Proof. Suppose $f$ and $g$ have an inessential coincidence class. Then from the proof of [[21], Theorem 5.1], it follows that $R(f, g)=\infty$ and that there exists $\bar{\alpha} \in A\left(N_{2}, \bar{p}_{2}\right)$ such that $R(\bar{\alpha} \bar{f}, \bar{g})=\infty$. So without loss of generality, we may assume that all coincidence 
classes are essential. Choose arbitrary $\alpha \in \Pi_{2}$. Then by assumption, $p_{1}(\operatorname{Coin}(\alpha \tilde{f}, \tilde{g}))$ is an essential coincidence class, where $p_{1}: G_{1} \rightarrow M_{1}$ is the natural covering projection. By Theorem 4.1 (3), it suffices to show that $\operatorname{coin}\left(\tau_{\alpha} \phi, \psi\right) \subset \Gamma_{1}$. In fact, $\operatorname{coin}\left(\tau_{\alpha} \phi\right.$, $\psi)=\{1\}$ by [[14], Lemma 4.8] together with the proof of [[14], Theorem 4.9].

\section{Averaging formula for the Lefschetz coincidence number}

The averaging formula for the Lefschetz coincidence number relates the Lefschetz coincidence number of a pair of continuous maps $f, g: M_{1} \rightarrow M_{2}$ to the Lefschetz coincidence numbers of lifts of $f$ and $g$ to finite sheeted regular covers of $M_{1}$ and $M_{2}$. The averaging formula for the Lefschetz coincidence number has been proved in [10] (see also [[9], p. 88]). In this section, we formulate a simple proof.

Remark 5.1 (See [[21], Lemma 3.9], [10] or [[22], p. 37]). Let $f, g: M_{1} \rightarrow M_{2}$ be continuous maps between closed oriented manifolds $M_{1}, M_{2}$ of equal dimension. Let $\bar{M}_{1}$ and $\bar{M}_{2}$ be covers of $M_{1}$ and $M_{2}$ respectively and suppose that the covering projections $\bar{p}_{1}: \bar{M}_{1} \rightarrow M_{1}$ and $\bar{p}_{2}: \bar{M}_{2} \rightarrow M_{2}$ are orientation preserving. Let $(\bar{f}, \bar{g}): \bar{M}_{1} \rightarrow \bar{M}_{2}$ be a lifting pair of $(f, g)$. Let $\bar{x} \in \operatorname{Coin}(\bar{f}, \bar{g})$. Then $x=p_{1}(\bar{x}) \in p_{1}(\operatorname{Coin}(\bar{f}, \bar{g})) \subset \operatorname{Coin}(f, g)$. Because the covering projections are orientation-preserving local diffeomorphisms,

$$
\text { ind }(f, g ;\{x\})=\text { ind }(\bar{f}, \bar{g} ;\{\bar{x}\}) \text {. }
$$

Theorem 5.2 (Averaging formula for the Lefschetz coincidence number). Let $p_{1}: \widetilde{M}_{1} \rightarrow M_{1}$ and $p_{2}: \widetilde{M}_{2} \rightarrow M_{2}$ be universal covers, let $\bar{p}_{2}: \bar{M}_{2} \rightarrow M_{2}$ and $(\bar{f}, \bar{g}): \bar{M}_{1} \rightarrow \bar{M}_{2}$ be finite sheeted, regular covers and let $p_{1}^{\prime}: \widetilde{M}_{1} \rightarrow \bar{M}_{1}$ and $p_{2}^{\prime}: \widetilde{M}_{2} \rightarrow \bar{M}_{2}$ be covers such that the diagrams

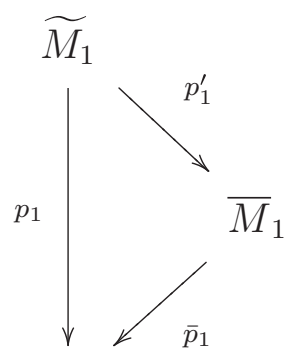

$M_{1}$

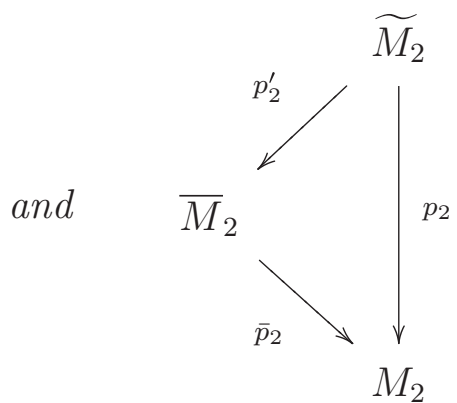

commute, where all spaces are connected oriented manifolds, where $\bar{M}_{1}$ and $\bar{M}_{2}$ are closed manifolds of equal dimension and where all covering projections are orientation preserving local diffeomorphisms. Suppose that $f$ has a lift $\bar{f}: \bar{M}_{1} \rightarrow \bar{M}_{2}$ and $g$ has a lift $\bar{g}: \bar{M}_{1} \rightarrow \bar{M}_{2}$. Then

$$
L(f, g)=\frac{1}{\# A\left(\bar{M}_{1}, \bar{p}_{1}\right)} \sum_{\bar{\alpha} \in A\left(\bar{M}_{2}, \bar{p}_{2}\right)} L(\bar{\alpha}, \bar{f}, \bar{g}) .
$$

Proof. It is well-known (cf. [[23], Theorem 3.1]) that there exists a pair of maps $\left(f, g^{\prime}\right)$ such that $(f, g)$ is homotopic to $\left(f^{\prime}, g^{\prime}\right)$ and $\operatorname{Coin}\left(f, g^{\prime}\right)$ is finite. Since the Lefschetz coincidence number is a homotopy invariant, we may assume that $\operatorname{Coin}(f, g)$ is finite. 
Because every $x \in \operatorname{Coin}(f, g)$ has $\# A\left(\bar{M}_{1}, \bar{p}_{1}\right)$ preimages under $\bar{p}_{1}$,

$$
\begin{aligned}
L(f, g) & =\sum_{x \in \operatorname{Coin}(f, g)} \operatorname{ind}(f, g ;\{x\}) \\
& =\frac{1}{\# A\left(\bar{M}_{1}, \bar{p}_{1}\right)} \sum_{\bar{x} \in p_{1}^{-1}(\operatorname{Coin}(f, g))} \operatorname{ind}\left(f, g ;\left\{\bar{p}_{1}(\bar{x})\right\}\right) .
\end{aligned}
$$

Now

$$
\bar{p}_{1}^{-1}(\operatorname{Coin}(f, g))=\coprod_{\bar{\alpha} \in A\left(\bar{M}_{2}, \bar{p}_{2}\right)} \operatorname{Coin}(\bar{\alpha} \bar{f}, \bar{g}) .
$$

Indeed, $\bar{x} \in \operatorname{Coin}(\bar{\alpha} \bar{f}, \bar{g}) \cap \operatorname{Coin}(\bar{\beta} \bar{f}, \bar{g})$ implies that $\bar{\alpha}(\bar{f}(\bar{x}))=\bar{g}(\bar{x})=\bar{\beta}(\bar{f}(\bar{x}))$, such that $\bar{\alpha}=\bar{\beta}$. Hence

$$
\begin{aligned}
L(f, g) & =\frac{1}{\# A\left(\bar{M}_{1}, \bar{p}_{1}\right)} \sum_{\bar{\alpha} \in A\left(\bar{M}_{2}, \bar{p}_{2}\right)} \sum_{\bar{x} \in \operatorname{Coin}(\bar{\alpha} f, \bar{g})} \text { ind }\left(f, g ;\left\{\bar{p}_{1}(\bar{x})\right\}\right) \\
& =\frac{1}{\# A\left(\bar{M}_{1}, \bar{p}_{1}\right)} \sum_{\bar{\alpha} \in A\left(\bar{M}_{2}, \bar{p}_{2}\right)} \sum_{\bar{x} \in \operatorname{Coin}(\bar{\alpha} f, \bar{g})} \text { ind }\left(\bar{\alpha} \bar{f}_{,}, \bar{g}_{;}\{\bar{x}\}\right)
\end{aligned}
$$

because of Remark 5.1. Hence

$$
\begin{aligned}
L(f, g) & =\frac{1}{\# A\left(\bar{M}_{1}, \bar{p}_{1}\right)} \sum_{\bar{\alpha} \in A\left(\bar{M}_{2}, \bar{p}_{2}\right)} \text { ind }(\bar{\alpha} \bar{f}, \bar{g} ; \operatorname{Coin}(\bar{\alpha} \bar{f}, \bar{g})) \\
& =\frac{1}{\# A\left(\bar{M}_{1}, \bar{p}_{1}\right)} \sum_{\bar{\alpha} \in A\left(\bar{M}_{2}, \bar{p}_{2}\right)} L(\bar{\alpha} \bar{f}, \bar{g}) .
\end{aligned}
$$

\section{Formulas for the Reidemeister, Lefschetz and Nielsen co-incidence number of maps between infra-nilmanifolds}

In this section, we give practical formulas for the Reidemeister coincidence number, the Lefschetz coincidence number and the Nielsen coincidence number of a pair of continuous maps between oriented infra-nilmanifolds of equal dimension. First we give some definitions and recall some useful results.

Definition 6.1. Let $G$ be an $n$-dimensional oriented, connected, simply connected, nilpotent Lie group and $\Lambda$ a uniform lattice in $G$. Let $\left\{a_{1}, \ldots, a_{n}\right\}$ be a set of generators of $\Lambda$ and write $b_{i}=\log a_{i}$, then $\left\{b_{1}, \ldots, b_{n}\right\}$ is a basis for the Lie algebra $\mathfrak{g}$ corresponding to $G$. Suppose additionally that the basis $\left\{b_{1}, \ldots, b_{n}\right\}$ is positively oriented. Then we refer to $\left\{b_{1}, \ldots, b_{n}\right\}$ as a preferred basis of $\Lambda$.

Definition 6.2. Let $V$ and $W$ be finite dimensional vector spaces and $L: V \rightarrow W$ a linear map. Let $\beta_{V}$ be a basis for $V$ and $\beta_{W}$ a basis for $W$, then we use $L_{\beta_{W}}^{\beta_{V}}$ to denote the matrix corresponding to $L$, where this matrix is expressed with respect to the bases $\beta_{V}$ and $\beta_{W}$.

Let $G_{1}$ and $G_{2}$ be oriented, connected, simply connected, nilpotent Lie groups of equal dimension with associated Lie algebras $\mathfrak{g}_{1}$ and $\mathfrak{g}_{2}$. Let $\Lambda_{1}$ be a uniform lattice in $G_{1}$ and $\Lambda_{2}$ a uniform lattice in $G_{2}$. Let $L: \mathfrak{g}_{1} \rightarrow \mathfrak{g}_{2}$ be a linear map. Let $\beta_{1}$ be a preferred basis of $\Lambda_{1}$ and $\beta_{2}$ a preferred basis of $\Lambda_{2}$. Then $\operatorname{det}\left(L_{\beta_{2}}^{\beta_{1}}\right)$ does not depend on 
the particular choice of the preferred bases $\beta_{1}$ of $\Lambda_{1}$ and $\beta_{2}$ of $\Lambda_{2}$ (see [[24], p. 253]). Hence we can define

$$
\stackrel{\Lambda_{1}}{\operatorname{det}_{2}}(L)=\operatorname{det}\left(L_{\beta_{2}}^{\beta_{1}}\right)
$$

for any choice of preferred bases $\beta_{1}$ of $\Lambda_{1}$ and $\beta_{2}$ of $\Lambda_{2}$.

The following theorem gives a formula for the Lefschetz coincidence number of a pair of continuous maps between nilmanifolds of equal dimension.

Theorem 6.3. [[13], Theorem 3.1] Let $G_{1}, G_{2}$ be oriented, connected, simply connected, nilpotent Lie groups of equal dimension. Let $\Lambda_{1}$ be a uniform lattice in $G_{1}$ and $\Lambda_{2}$ a uniform lattice in $G_{2}$. Let $N_{1}=\Lambda_{1} \backslash G_{1}$ and $N_{2}=\Lambda_{2} \backslash G_{2}$ be the corresponding nilmanifolds. Let $f, f^{\prime}: N_{1} \rightarrow N_{2}$ be continuous maps. Let $D, D^{\prime}: G_{1} \rightarrow G_{2}$ be morphisms of Lie groups such that $D$ is a homotopy lift of $f$ and $D^{\prime}$ is a homotopy lift of $f$. Then

$$
L\left(f, f^{\prime}\right)=\operatorname{det}_{\Lambda_{2}}^{\Lambda_{1}}\left(D_{*}^{\prime}-D_{*}\right) .
$$

Remark that Theorem 2.7 guarantees the existence of morphisms of Lie groups $D, D^{\prime}$ $: G_{1} \rightarrow G_{2}$ that are homotopy lifts of $f, f: N_{1} \rightarrow N_{2}$.

Lemma 6.4. [[24], Lemma 3.2] Let $G$ be an oriented, connected, simply connected, nilpotent Lie group and $\Lambda$ a uniform lattice in G. Let $\hat{\Lambda}$ be a finite index subgroup of $\Lambda$. Then

$$
\operatorname{det}_{\Lambda}^{\hat{\Lambda}}(\mathrm{Id})=[\Lambda: \hat{\Lambda}]
$$

Proof. Define $N=\Lambda \backslash G$ and $\hat{N}=\hat{\Lambda} \backslash G$ and give $N$ and $\hat{N}$ orientations so that the covering projections $G \rightarrow N$ and $G \rightarrow \hat{N}$ are orientation preserving. Then Id $G \rightarrow G: g$ $\mapsto g$ induces an orientation preserving map $f^{\prime}: \hat{N} \rightarrow N$ and $O: G \rightarrow G: g \mapsto 1_{G}$ induces a constant map $f: \hat{N} \rightarrow N$. By Theorem 6.3,

$$
L\left(f, f^{\prime}\right)=\operatorname{det}_{\Lambda}^{\hat{\Lambda}}(\mathrm{Id}) .
$$

On the other hand, by [[7], Lemma 3.10], every essential coincidence class is a singleton and has coincidence index $\operatorname{sign}\left(\operatorname{det}_{\Lambda}^{\hat{\Lambda}}(\mathrm{Id})\right)$. Remark that sign $\operatorname{sign}\left(\operatorname{det}_{\Lambda}^{\hat{\Lambda}}(\mathrm{Id})\right)=1$. So it suffices to show that $\# \operatorname{Coin}\left(f, f^{\prime}\right)=[\Lambda: \hat{\Lambda}]$. Now

$$
\begin{aligned}
\operatorname{Coin}\left(f, f^{\prime}\right) & =\left\{\hat{\Lambda} g \in \hat{N} \mid f(\hat{\Lambda} g)=f^{\prime}(\hat{\Lambda} g)\right\} \\
& =\{\hat{\Lambda} g \in \hat{N} \mid \Lambda g=\Lambda\} \\
& =\{\hat{\Lambda} g \in \hat{N} \mid g=\Lambda\} .
\end{aligned}
$$

Hence \#Coin $\left(f, f^{\prime}\right)=[\Lambda: \hat{\Lambda}]$.

Corollary 6.5. Let $G$ be an oriented, connected, simply connected, nilpotent Lie group and $\Lambda$ a uniform lattice in G. Let $\hat{\Lambda}$ be a finite index subgroup of $\Lambda$. Then 


$$
\operatorname{det}_{\Lambda}^{\hat{\Lambda}}(\mathrm{Id})=\frac{1}{[\Lambda: \hat{\Lambda}]}
$$

Proof. Let $\beta$ be a preferred basis of $\Lambda$ and $\hat{\beta}$ a preferred basis of $\hat{\Lambda}$. Then

$$
\operatorname{det}_{\Lambda}^{\hat{\Lambda}}(\mathrm{Id}) \operatorname{det}_{\hat{\Lambda}}^{\Lambda}(\mathrm{Id})=\operatorname{det}\left(\operatorname{Id}_{\beta}^{\hat{\beta}} \operatorname{Id}_{\hat{\beta}}^{\beta}\right)=\operatorname{det}\left(\operatorname{Id}_{\beta}^{\beta}\right)=1
$$

and the corollary follows from the previous lemma.

Corollary 6.6. Let $G_{1}$ and $G_{2}$ be oriented, connected, simply connected, nilpotent Lie groups with associated Lie algebras $\mathfrak{g}_{1}$ and $\mathfrak{g}_{2}$. Let $\Lambda_{1}$ be a uniform lattice of $G_{1}$ and $\Lambda_{2}$ a uniform lattice of $G_{2}$. Let $\hat{\Lambda}_{1}$ be a finite index subgroup of $\Lambda_{1}$ and $\hat{\Lambda}_{2}$ a finite index subgroup of $\Lambda_{2}$. Then for any linear map $L: \mathfrak{g}_{1} \rightarrow \mathfrak{g}_{2}$,

$$
\underset{\hat{\Lambda}_{2}}{\hat{\operatorname{det}}_{1}}(L)=\frac{\left[\Lambda_{1}: \hat{\Lambda}_{1}\right]}{\left[\Lambda_{2}: \hat{\Lambda}_{2}\right]} \underset{\Lambda_{2}}{\operatorname{det}}(L) .
$$

Proof. This follows from a short calculation:

$$
\underset{\hat{\Lambda}_{2}}{\hat{\Lambda}_{1}}(L)=\operatorname{det}_{\hat{\Lambda}_{2}}^{\Lambda_{2}}(\text { Id }) \underset{\Lambda_{2}}{\operatorname{det}_{1}}(L) \underset{\Lambda_{1}}{\hat{\Lambda}_{1}}(\text { Id })=\frac{\left[\Lambda_{1}: \hat{\Lambda}_{1}\right]}{\left[\Lambda_{2}: \hat{\Lambda}_{2}\right]} \operatorname{det}(L) .
$$

In order to prove the main formulas of this article, we will prove the following technical theorem, which generalizes [[12], Lemma 3.2].

Theorem 6.7. Let $G_{1}$ and $G_{2}$ be connected, simply connected, nilpotent Lie groups of equal dimension with associated Lie algebras $\mathfrak{g}_{1}$ and $\mathfrak{g}_{2}$ respectively. Let $D, D^{\prime}: G_{1} \rightarrow$ $G_{2}$ be morphisms of Lie groups inducing morphisms of Lie algebras $D_{*}, D_{*}^{\prime}: \mathfrak{g}_{1} \rightarrow \mathfrak{g}_{2}$. Then for any $g \in G_{2}$,

$$
\operatorname{det}\left(D_{*}^{\prime}-D_{*}\right)=\operatorname{det}\left(D^{\prime}{ }_{*}-\operatorname{Ad}(g) D_{*}\right) .
$$

The proof of this theorem expresses the right hand side of the equality as a polynomial. Then we use the following lemma to prove that either this polynomial is the zero polynomial or it has no roots.

Lemma 6.8. [[21], Lemma 3.5] Let $G_{1}$ and $G_{2}$ be connected, simply connected, nilpotent (complex) Lie groups with associated (complex) Lie algebras $\mathfrak{g}_{1}$ and $\mathfrak{g}_{2}$ respectively. Let $D, D^{\prime}: G_{1} \rightarrow G_{2}$ be morphisms of Lie groups inducing morphisms of Lie algebras $D_{*}, D_{*}^{\prime}: \mathfrak{g}_{1} \rightarrow \mathfrak{g}_{2}$. Then for any $g \in G_{2}, D_{*}^{\prime}-D_{*}$ is surjective if and only if $D_{*}^{\prime}-\operatorname{Ad}(g) D_{*}$ is surjective.

In fact, this lemma was only proved in the real case, but its proof is also valid in the complex case. Now we can prove Theorem 6.7.

Proof of Theorem 6.7. Let $G_{1}^{\mathbb{C}}$ and $G_{2}^{\mathbb{C}}$ be the complexifications of the real Lie groups $G_{1}$ and $G_{2}$ respectively with canonical maps $\alpha_{i}: G_{i} \rightarrow G_{i}^{\mathbb{C}}$. Since $G_{i}$ is simply connected, $\alpha_{i}$ is injective. Let $\mathfrak{g}_{i}$ and $\mathfrak{g}_{i}^{\mathbb{C}}$ denote the respective Lie algebras. Then $\mathfrak{g}_{i}^{\mathbb{C}} \cong \mathfrak{g}_{i} \otimes_{\mathbb{R}} \mathbb{C}$. 
The morphism $D: G_{1} \rightarrow G_{2}$ extends uniquely to a morphism $D^{\mathbb{C}}: G_{1}^{\mathbb{C}} \rightarrow G_{2}^{\mathbb{C}}$ of complex Lie groups so that $\left(D^{\mathbb{C}}\right)_{*}=D_{*}^{\mathbb{C}}$. Similarly, we define $D^{\prime \mathbb{C}}: G_{1}^{\mathbb{C}} \rightarrow G_{2}^{\mathbb{C}}, D^{\prime}{ }_{*}^{\mathbb{C}}: \mathfrak{g}_{1}^{\mathbb{C}} \rightarrow \mathfrak{g}_{2}^{\mathbb{C}}$ and $\operatorname{Ad}(g)^{\mathbb{C}}$ for any $g \in G_{2}$.

Let $\left\{X_{1}^{1}, \ldots, X_{1}^{n}\right\}$ be a basis for $\mathfrak{g}_{1}$ and $\left\{X_{2}^{1}, \ldots, X_{2}^{n}\right\}$ a basis for $\mathfrak{g}_{2}$. Write $Y_{1}^{i}=\left(\alpha_{1}\right)_{*}\left(X_{1}^{i}\right)$ and $Y_{2}^{i}=\left(\alpha_{2}\right)_{*}\left(X_{2}^{i}\right)$, then $\left\{Y_{1}^{1} \ldots, Y_{1}^{n}\right\}$ is a basis for the complex vector space $\mathfrak{g}_{1}^{\mathbb{C}}$ and $\left\{Y_{2}^{1} \ldots, Y_{2}^{n}\right\}$ is a basis for the complex vector space $\mathfrak{g}_{2}^{\mathbb{C}}$. Now

$$
\operatorname{det}\left(D^{\prime} *-\operatorname{Ad}(g) D_{*}\right)=\operatorname{det}\left(D_{*}^{\mathbb{C}}-\operatorname{Ad}\left(\alpha_{2}(g)\right) D_{*}^{\mathbb{C}}\right),
$$

where on the left hand side, the matrices are expressed with respect to the bases $\left\{X_{1}^{1}, \ldots, X_{1}^{n}\right\}$ and $\left\{X_{2}^{1}, \ldots, X_{2}^{n}\right\}$ and on the right hand side, the matrices are expressed with respect to the bases $\left\{Y_{1}^{1} \ldots, Y_{1}^{n}\right\}$ and $\left\{Y_{2}^{1} \ldots, Y_{2}^{n}\right\}$.

Now we consider the function $f: \mathfrak{g}_{2}^{\mathbb{C}} \rightarrow \mathbb{C}$ defined by

$$
f(Y)=\operatorname{det}\left(D_{*}^{\mathbb{C}}-\operatorname{Ad}(\exp (Y)) D_{*}^{\mathbb{C}}\right) .
$$

By Lemma 6.8, either $f$ is the zero map or $f$ has no roots.

Choose arbitrary $Y \in \mathfrak{g}_{2}^{\mathbb{C}}$. With respect to the complex basis $\left\{Y_{2}^{1} \ldots, Y_{2}^{n}\right\}$ of $\mathfrak{g}_{2}^{\mathbb{C}} \cdot \operatorname{ad}(Y)$ is regarded as a matrix $\left[z_{i j}(Y)\right]$ where all $z_{i j}: \mathfrak{g}_{2}^{\mathbb{C}} \rightarrow \mathbb{C}$ are complex-valued functions. Now there exist complex numbers $c_{i}^{k j}$ such that

$$
\left[Y_{2}^{k}, Y_{2}^{j}\right]=\sum_{i} c_{i}^{k j} Y_{2}^{i}
$$

Writing $Y=\sum_{k} \lambda_{k} Y_{2}^{k}$, we remark that

$$
\begin{aligned}
\sum_{i} z_{i j}(Y) Y_{2}^{i} & =\operatorname{ad}(Y)\left(Y_{2}^{i}\right)=\left[Y, Y_{2}^{j}\right]=\sum_{k} \lambda_{k}\left[Y_{2}^{k}, Y_{2}^{j}\right] \\
& =\sum_{k} \sum_{i} \lambda_{k} c_{i}^{k j} Y_{2}^{i}=\sum_{i}\left(\sum_{k} \lambda_{k} c_{i}^{k j}\right) Y_{2}^{i} .
\end{aligned}
$$

So the entries $z_{i j}(Y)=\sum_{k} c_{i}^{k j} \lambda_{k}$ of $\operatorname{ad}(Y)$ depend linearly on the $\lambda_{k}$, and hence the entries of $\operatorname{Ad}(\exp (Y))=\exp (\operatorname{ad}(Y))$ depend polynomially on the $\lambda_{k}$. Consequently,

$$
f(Y)=\operatorname{det}\left(D_{*}^{\mathbb{C}}-\exp (\operatorname{ad}(Y)) D_{*}^{\mathbb{C}}\right)
$$

is a polynomial in $Y$.

If $f$ is not the zero map, then $f$ has no roots and from the fundamental theorem of algebra, it follows that $f$ is a constant polynomial. Hence, regardless of whether $f$ is the zero map or not, $f$ is constant and for any $g \in G_{2}$,

$$
\operatorname{det}\left(D^{\prime} *-\operatorname{Ad}(g) D_{*}\right)=\operatorname{det}\left(D_{*}{ }_{*}^{\mathbb{C}}-\operatorname{Ad}\left(\alpha_{2}(g)\right) D_{*}^{\mathbb{C}}\right)=f\left(\log \left(\alpha_{2}(g)\right)\right)
$$

does not depend on $g$. This proves the theorem.

Now we generalize [[12], Lemma 3.1], in which the existence of a fully invariant subgroup of finite index in an almost-Bieberbach group is proved. The proof consists 
merely of a straightforward adaptation of that of [[12], Lemma 3.1] to this more general, but very analogous situation.

Lemma 6.9. Let $\Pi_{1}$ and $\Pi_{2}$ be almost-crystallographic groups and let $\Gamma_{i}$ be the maximal normal nilpotent subgroup of $\Pi_{i}$ or, more generally, let $\Pi_{i} \subset S_{i} \rtimes \operatorname{Aut}\left(S_{i}\right)$ be a finite extension of the lattice $\Gamma_{i}$ of a connected, and simply connected solvable Lie group $S_{i}$. Then there exist fully invariant subgroups $\Lambda_{i} \subset \Gamma_{i}$ of $\Pi_{i}$, which are of finite index, so that any morphism $\Pi_{1} \rightarrow \Pi_{2}$ maps $\Lambda_{1}$ into $\Lambda_{2}$.

Proof. Let $k$ be the least common multiple of the orders of the holonomy groups $\Pi_{1} /$ $\Gamma_{1}$ and $\Pi_{2} / \Gamma_{2}$. Let $\Lambda_{1}$ be the subgroup of $\Pi_{1}$ generated by the set

$$
\left\{x^{k} \mid x \in \prod_{1}\right\} .
$$

Clearly, the generating set is a subset of $\Gamma_{1}$ so that $\Lambda_{1}$ is a subgroup of $\Gamma_{1}$. Similarly, the subgroup $\Lambda_{2}$ of $\Pi_{2}$ generated by $\left\{y^{k} \mid y \in \Pi_{2}\right\}$ is a subgroup of $\Gamma_{2}$. Obviously, any morphism $\theta: \Pi_{1} \rightarrow \Pi_{2}$ sends the generating set $\left\{x^{k} \mid x \in \Pi_{1}\right\}$ of $\Lambda_{1}$ into the generating set $\left\{y^{k} \mid y \in \Pi_{2}\right\}$ of $\Lambda_{2}$. Thus $\theta$ maps $\Lambda_{1}$ into $\Lambda_{2}$.

We claim that $\Lambda_{1}$ has finite index in $\Gamma_{1}$ (and hence in $\Pi_{1}$ ). Consider the subgroup $\Gamma(k)$ generated by the set $\left\{x^{k} \mid x \in \Gamma_{1}\right\}$. Since $\Gamma_{1}$ is a lattice in the connected and simply connected solvable Lie group $S_{1}$, it is a (strongly) polycyclic group. Then $\Gamma(k)$ has finite index in $\Gamma_{1}$, see [[25], Lemma 4.4]. Since $\Gamma(k) \subset \Lambda_{1}$, we find that $\Lambda_{1}$ has finite index in $\Gamma_{1}$. Similarly, $\Lambda_{2}$ has finite index in $\Gamma_{2}$ and hence in $\Pi_{2}$.

By taking $S_{1}=S_{2}$ and $\Pi_{1}=\Pi_{2}$, we see that any morphism on $\Pi_{i}$ maps $\Lambda_{i}$ into $\Lambda_{i}$ itself. Hence $\Lambda_{i}$ is a fully invariant subgroup of $\Pi_{i}$.

Let us recall the averaging formula for the Nielsen coincidence number in the special case of maps between infra-nilmanifolds.

Theorem 6.10. [[14], Theorem 4.9] Let $M_{1}$ and $M_{2}$ be closed oriented infra-nilmanifolds of equal dimension and $f, g: M_{1} \rightarrow M_{2}$ continuous maps. Suppose there exist finite sheeted regular covers $\bar{p}_{1}: N_{1} \rightarrow M_{1}$ and $\bar{p}_{2}: N_{2} \rightarrow M_{2}$, where $N_{1}$ and $N_{2}$ are nilmanifolds. Suppose that $\bar{f}: N_{1} \rightarrow N_{2}$ is a lift of $f$ and $\bar{g}: N_{1} \rightarrow N_{2}$ is a lift of $g$. Then

$$
N(f, g)=\frac{1}{\# A\left(N_{1}, \bar{p}_{1}\right)} \sum_{\bar{\alpha} \in A\left(N_{2}, \bar{p}_{2}\right)} N(\bar{\alpha} \bar{f}, \bar{g}) .
$$

Now we prove practical formulas for the Reidemeister coincidence number, the Lefschetz coincidence number and the Nielsen coincidence number of a pair of continuous maps between oriented infra-nilmanifolds of equal dimension.

Theorem 6.11. Let $G_{1}$ and $G_{2}$ be connected, simply connected, nilpotent Lie groups of equal dimension. Let $\Pi_{1}$ and $\Pi_{2}$ be almost-Bieberbach groups modeled on $G_{1}$ and $G_{2}$, respectively and suppose that the corresponding infra-nilmanifolds $M_{1}=\Pi_{1} \backslash G_{1}$ and $M_{2}$ $=\Pi_{2} \backslash G_{2}$ are oriented. Let $F_{1} \subset \operatorname{Aut}\left(G_{1}\right)$ be the holonomy group of $M_{1}$ and $F_{2} \subset \operatorname{Aut}\left(G_{2}\right)$ the holonomy group of $M_{2}$. Let $f, g: M_{1} \rightarrow M_{2}$ be continuous maps. Let $D, D^{\prime}: G_{1} \rightarrow G_{2}$ be morphisms of Lie groups and $d, d^{\prime} \in G_{2}$ such that $\lambda_{d} \circ D: G_{1} \rightarrow G_{2}$ is a homotopy lift off and $\lambda_{d^{\prime}} \circ D^{\prime}: G_{1} \rightarrow G_{2}$ is a homotopy lift of $g$. (Recall that $\lambda_{d}: G_{2} \rightarrow G_{2}: g^{\prime} \mapsto d g^{\prime}$ is the left multiplication map.) Then 


$$
\begin{aligned}
& L(f, g)=\frac{1}{\# F_{1}} \sum_{A \in F_{2}} \operatorname{det}\left(D^{\prime}{ }_{*}-A_{*} D_{*}\right), \\
& N(f, g)=\frac{1}{\# F_{1}} \sum_{A \in F_{2}}\left|\operatorname{det}\left(D^{\prime}{ }_{*}-A_{*} D_{*}\right)\right|,
\end{aligned}
$$

and

$$
R(f, g)=\frac{1}{\# F_{1}} \sum_{A \in F_{2}} \sigma\left(\operatorname{det}\left(D_{*}^{\prime}-A_{*} D_{*}\right)\right),
$$

where the morphisms of Lie groups $D^{*}, D_{*}^{\prime}$ and $A_{*}$ induced by $D, D^{\prime}$ and $A$ are expressed with respect to preferred bases of $\Pi_{1} \cap G_{1}$ and $\Pi_{2} \cap G_{2}$ and where $\sigma: \mathbb{R} \rightarrow \mathbb{R}$ $\mathrm{U}\{\infty\}$ is defined by $\sigma(0)=\infty$ and $\sigma(x)=|x|$ for all $x \neq 0$.

Remark that by Theorem 2.6, there exist $d, d^{\prime} \in G_{2}$ and morphisms of Lie groups $D$, $D^{\prime}: G_{1} \rightarrow G_{2}$ such that $\lambda_{d} \circ D: G_{1} \rightarrow G_{2}$ is a homotopy lift of $f$ and $\lambda_{d^{\prime}} \circ D^{\prime}: G_{1} \rightarrow$ $G_{2}$ is a homotopy lift of $g$.

Proof of Theorem 6.11. Without loss of generality, we may assume that $\lambda_{d} \circ D$ is a lift of $f$ and $\lambda_{d^{\prime}} \circ D^{\prime}$ is a lift of $g$. Define $\Gamma_{1}=\Pi_{1} \cap G_{1}$ and $\Gamma_{2}=\Pi_{2} \cap G_{2}$. By Lemma 6.9, there exist uniform lattices $\Lambda_{1} \subset G_{1}$ and $\Lambda_{2} \subset G_{2}$ such that $\Lambda_{i}$ is a fully invariant subgroup of $\Pi_{i}, \Lambda_{i}$ is a finite index subgroup of $\Gamma_{i}$ and such that $\theta\left(\Lambda_{1}\right) \subset \Lambda_{2} \subset \Gamma_{2}$ for every morphism of groups $\theta: \Pi_{1} \rightarrow \Pi_{2}$. Define $N_{1}=\Lambda_{1} \backslash G_{1}$ and $N_{2}=\Gamma_{2} \backslash G_{2}$. Let $\bar{p}_{2}: N_{2} \rightarrow M_{2}, \bar{p}_{2}: N_{2} \rightarrow M_{2}, p_{1}^{\prime}: G_{1} \rightarrow N_{1}$ and $p_{2}^{\prime}: G_{2} \rightarrow N_{2}$ be natural projections. Then $f, g$ lift to continuous maps $\bar{f}, \bar{g}: N_{1} \rightarrow N_{2}$ so that the following diagram commutes up to homotopy

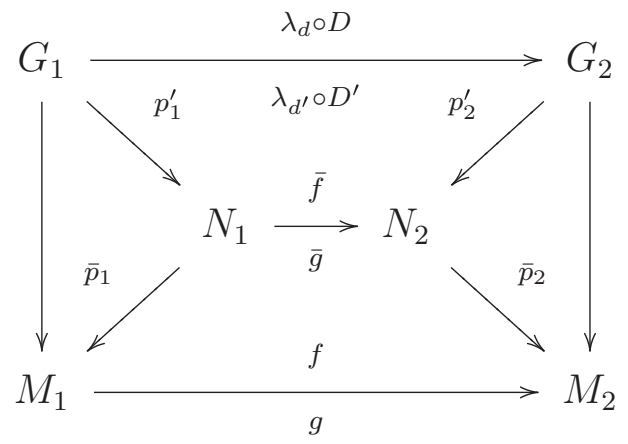

By Theorem 5.2, the averaging formula for the Lefschetz coincidence number,

$$
L(f, g)=\frac{1}{\left[\prod_{1}: \Lambda_{1}\right]} \sum_{\bar{\alpha} \in A\left(N_{2}, \bar{p}_{2}\right)} L(\bar{\alpha} \bar{f}, \bar{g}) .
$$

By [[9], Theorem 1.1], $N(\bar{\alpha} \bar{f}, \bar{g})=|L(\bar{\alpha} \bar{f}, \bar{g})|$ for every $\bar{\alpha} \in A\left(N_{2}, \bar{p}_{2}\right)$. Hence by Theorem 6.10, the averaging formula for the Nielsen coincidence number,

$$
\begin{aligned}
N(f, g) & =\frac{1}{\left[\prod_{1}: \Lambda_{1}\right]} \sum_{\bar{\alpha} \in A\left(N_{2}, \bar{p}_{2}\right)} N(\bar{\alpha} \bar{f}, \bar{g}) \\
& =\frac{1}{\left[\prod_{1}: \Lambda_{1}\right]} \sum_{\bar{\alpha} \in A\left(N_{2}, \bar{p}_{2}\right)}|L(\bar{\alpha} \bar{f}, \bar{g})| .
\end{aligned}
$$


By the main result of [26], $R(\bar{\alpha} \bar{f}, \bar{g})=\sigma(L(\bar{\alpha} \bar{f}, \bar{g}))$ for every $\bar{\alpha} \in A\left(N_{2}, \bar{p}_{2}\right)$. Hence by Theorem 4.2, the averaging formula for the Reidemeister coincidence number,

$$
\begin{aligned}
R(f, g) & =\frac{1}{\left[\prod_{1}: \Lambda_{1}\right]} \sum_{\bar{\alpha} \in A\left(N_{2}, \bar{p}_{2}\right)} R(\bar{\alpha} \bar{f}, \bar{g}) \\
& =\frac{1}{\left[\prod_{1}: \Lambda_{1}\right]} \sum_{\bar{\alpha} \in A\left(N_{2}, \bar{p}_{2}\right)} \sigma(L(\bar{\alpha} \bar{f}, \bar{g})) .
\end{aligned}
$$

Choose arbitrary $\bar{\alpha} \in A\left(N_{2}, \bar{p}_{2}\right)$. Then there exist $a \in G_{2}$ and $A$ in the holonomy group $F_{2} \subset \operatorname{Aut}\left(G_{2}\right)$ such that $\lambda_{a} \circ A: G_{2} \rightarrow G_{2}$ is a lift of $\bar{\alpha}$. We now claim that

$$
L(\bar{\alpha} \bar{f}, \bar{g})=\left[\Gamma_{1}: \Lambda_{1}\right] \operatorname{\Gamma et}_{\Gamma_{2}}^{\Gamma_{1}}\left(D_{*}^{\prime}-A_{*} D_{*}\right) .
$$

First remark that $\bar{f}: N_{1} \rightarrow N_{2}$ induces a morphism $\bar{f}_{\times}: A\left(G_{1}, p_{i}^{\prime}\right) \rightarrow A\left(G_{2}, p_{2}{ }_{2}\right)$ defined by

$$
\bar{f}_{\times}(\lambda) \circ\left(\lambda_{d} \circ D\right)=\left(\lambda_{d} \circ D\right) \circ \lambda \quad \text { for every } \lambda \in A\left(G_{1}, p_{1}^{\prime}\right) .
$$

If we apply both sides of this equality to the identity element $1_{G_{1}}$ of $G_{1}$, then we see that $\bar{f}_{\times}(\lambda)=\left(\tau_{d} \circ D\right)(\lambda)$. Hence $\left(\tau_{d} \circ D\right)\left(\Lambda_{1}\right) \subset \Gamma_{2}$ and $\tau_{d} \circ D: G_{1} \rightarrow G_{2}$ induces a continuous map $h: N_{1} \rightarrow N_{2}$. A similar calculation shows that $h_{\times}=\tau_{d} \circ D=\bar{f}_{\times}$. Because $N_{1}$ and $N_{2}$ are aspherical, $\bar{f}$ and $h$ are homotopic and we see that $\tau_{d} \circ D$ is a homotopy lift of $\bar{f}$.

Similarly, one can show that $\tau_{d^{\prime}} \circ D^{\prime}$ is a homotopy lift of $\bar{g}$ and that $\tau_{a} \circ A$ is a homotopy lift of $\bar{\alpha}$. Then by Theorem 6.3,

$$
\begin{aligned}
L(\bar{\alpha} \bar{f}, \bar{g}) & =\operatorname{det}_{\Gamma_{2}}^{\Lambda_{1}}\left(\left(\tau_{d^{\prime}} \circ D^{\prime}\right)_{*}-\left(\tau_{a} \circ A \circ \tau_{d} \circ D\right)_{*}\right) \\
& =\operatorname{det}_{\Gamma_{2}}\left(\left(\tau_{d^{\prime}}\right)_{*} D_{*}^{\prime}-\left(\tau_{a A(d)}\right)_{*} A_{*} D_{*}\right) .
\end{aligned}
$$

By applying Theorem 6.7 twice, we see that

$$
L(\bar{\alpha} \bar{f}, \bar{g})=\operatorname{det}_{\Gamma_{2}}^{\Lambda_{1}}\left(\left(\tau_{d^{\prime}}\right)_{*} D^{\prime}{ }_{*}-\left(\tau_{a A(d)}\right)_{*} A_{*} D_{*}\right)=\operatorname{det}_{\Gamma_{2}}^{\Lambda_{1}}\left(D^{\prime}{ }_{*}-A_{*} D_{*}\right) .
$$

By Corollary 6.6,

$$
L(\bar{\alpha} \bar{f}, \bar{g})=\operatorname{det}_{\Gamma_{2}}^{\Lambda_{1}}\left(D^{\prime}{ }_{*}-A_{*} D_{*}\right)=\left[\Gamma_{1}: \Lambda_{1}\right] \operatorname{det}_{\Gamma_{2}}^{\Gamma_{1}}\left(D^{\prime}{ }_{*}-A_{*} D_{*}\right) .
$$

Since $\bar{\alpha}$ was chosen arbitrarily, this equality holds for every $\bar{\alpha}$, where $A \in F_{2}$ is such that $\lambda_{a} \circ A$ is a lift of $\bar{\alpha}$ for some $a \in G_{2}$. Hence

$$
\begin{aligned}
L(f, g) & =\frac{1}{\left[\Pi_{1}: \Lambda_{1}\right]} \sum_{\bar{\alpha} \in A\left(N_{2}, \bar{p}_{2}\right)} L(\bar{\alpha}, \bar{f}, \bar{g}) \\
& =\frac{1}{\left[\Pi_{1}: \Lambda_{1}\right]} \sum_{A \in F_{2}}\left[\Gamma_{1}: \Lambda_{1}\right] \underset{\Gamma_{2}}{\Gamma_{1}}\left(D^{\prime}{ }^{\prime}-A_{*} D_{*}\right) \\
& =\frac{1}{\left[\Pi_{1}: \Gamma_{1}\right]} \sum_{A \in F_{2}} \operatorname{det}_{\Gamma_{2}}^{\Gamma_{1}}\left(D \prime_{*}-A_{*} D_{*}\right) \\
& =\frac{1}{\# F_{1}} \sum_{A \in F_{2}} \operatorname{det}_{\Gamma_{2}}^{\operatorname{det}_{1}}\left(D \prime_{*}-A_{*} D_{*}\right) .
\end{aligned}
$$


Similar calculations show that

$$
N(f, g)=\frac{1}{\# F_{1}} \sum_{A \in F_{2}}\left|\underset{\Gamma_{2}}{\operatorname{det}}\left(D_{*}^{\prime}{ }_{*}-A_{*} D_{*}\right)\right|
$$

and that

$$
R(f, g)=\frac{1}{\# F_{1}} \sum_{A \in F_{2}} \sigma\left(\underset{\Gamma_{2}}{\Gamma_{1}}\left(D_{*}^{\prime}-A_{*} D_{*}\right)\right) .
$$

The following generalizes [[8], Theorem 2.2] from the fixed point version to the coincidence version.

Corollary 6.12. Let $G_{1}$ and $G_{2}$ be connected, simply connected, nilpotent Lie groups of equal dimension. Let $M_{1}$ and $M_{2}$ be oriented infra-nilmanifolds modeled on $G_{1}$ and $G_{2}$ respectively. Let $F_{2} \subset \operatorname{Aut}\left(G_{2}\right)$ be the holonomy group of $M_{2}$. Let $f, g: M_{1} \rightarrow M_{2}$ be continuous maps. Let $D, D^{\prime}: G_{1} \rightarrow G_{2}$ be morphisms of Lie groups and $d$, d' $\in G_{2}$ such that $\lambda_{d} \circ D: G_{1} \rightarrow G_{2}$ is a homotopy lift of $f$ and $\lambda_{d}{ }^{\circ} \circ D^{\prime}: G_{1} \rightarrow G_{2}$ is a homotopy lift of $g$. Then $N(f, g)=L(f, g)$ if and only if $\operatorname{det}\left(D^{\prime}{ }_{*}-A_{*} D_{*}\right) \geq 0$ for every $A \in F_{2}$ and $N(f, g)$ $=-L(f, g)$ if and only if $\operatorname{det}\left(D_{*}^{\prime}-A_{*} D_{*}\right) \leq 0$ for every $A \in F_{2}$, where $D_{*}, D_{*}^{\prime}$ and $A_{*}$ are the morphisms of Lie algebras induced by D, D' and A respectively, expressed with respect to positively oriented bases of the Lie algebras associated to $G_{1}$ and $G_{2}$.

\section{Examples}

In this section we illustrate, by some examples, how practical the averaging formulas on infra-nilmanifolds are. For this purpose we will consider maps from a 3-dimensional flat Riemannian manifold to an infra-nilmanifold modeled on the Heisenberg group Nil.

Let Nil be the 3-dimensional Heisenberg group defined by

$$
\text { Nil }=\left\{\left[\begin{array}{lll}
1 & x & z \\
0 & 1 & y \\
0 & 0 & 1
\end{array}\right] \mid x, y, z \in \mathbb{R}\right\} .
$$

Then it is a connected and simply connected 2-step nilpotent Lie group. The corresponding Lie algebra is

$$
\mathfrak{n i l}=\left\{\left[\begin{array}{lll}
0 & x & z \\
0 & 0 & y \\
0 & 0 & 0
\end{array}\right] \mid x, y, z \in \mathbb{R}\right\} .
$$

It is easy to see that (cf. [[27], Proposition 2.2])

$$
\operatorname{Aut}(\mathrm{Nil})=\left\{\left[\begin{array}{cc}
\operatorname{det}(A) & p \\
0 & A
\end{array}\right] \mid A \in \mathrm{GL}(2, \mathbb{R}), \mathrm{p} \in \mathbb{R}^{2} \text { is a row vector }\right\}
$$

and an element

$$
\left[\begin{array}{cc}
\operatorname{det}(A) & \mathrm{p} \\
0 & A
\end{array}\right] \in \operatorname{Aut}(\mathrm{Nil}) \text { with } \mathrm{p}=[u v], A=\left[\begin{array}{ll}
p & q \\
r & s
\end{array}\right]
$$


acts on Nil as follows:

$$
\left[\begin{array}{lll}
1 & x & z \\
0 & 1 & y \\
0 & 0 & 1
\end{array}\right] \mapsto\left[\begin{array}{ccc}
1 & p x+q y & z^{\prime} \\
0 & 1 & r x+s y \\
0 & 0 & 1
\end{array}\right]
$$

where

$$
z^{\prime}=(p s-q r) z+\frac{1}{2}\left(p r x^{2}+2 q r x y+q s y^{2}\right)+u x+v y
$$

Example 7.1. Let $\Pi_{1}$ be the Bieberbach group generated by the standard basis $\left\{\mathbf{e}_{1}, \mathbf{e}_{2}\right.$, $\left.\mathbf{e}_{3}\right\}$ of $\mathbb{R}^{3}$. Let $M_{1}=\Pi_{1} \backslash \mathbb{R}^{3}$ be the corresponding infra-nilmanifold, the 3 -dimensional flat torus, with the trivial holonomy group.

Consider the almost Bieberbach group $\Pi_{2}$ given by

$$
\Pi_{2}=\left\langle\begin{array}{l|l}
s_{1}, s_{2}, s_{3}, \alpha \mid \begin{array}{c}
{\left[s_{2}, s_{1}\right]=s_{3}^{2},\left[s_{3}, s_{1}\right]=\left[s_{3}, s_{2}\right]=1,} \\
\alpha^{2}=s_{1} \alpha s_{1} \alpha^{-1}=s_{1}, \alpha s_{2}=s_{2}^{-1} \alpha s_{3}^{-1}
\end{array}
\end{array}\right\rangle .
$$

This is a 3-dimensional orientable almost Bieberbach group $\pi_{3}$ with Seifert bundle type 3 ( [[28], Proposition 6.1], or the list of [[29], p. 800]). We can embed $\Pi_{2}$ into Aff $(\mathrm{Nil})=\mathrm{Nil} \rtimes$ Aut $(\mathrm{Nil})$ by taking

$$
\begin{aligned}
& s_{1}=\left(\left[\begin{array}{lll}
1 & 1 & 0 \\
0 & 1 & 0 \\
0 & 0 & 1
\end{array}\right], I\right), s_{2}=\left(\left[\begin{array}{lll}
1 & 0 & 0 \\
0 & 1 & 1 \\
0 & 0 & 1
\end{array}\right], I\right), \\
& s_{3}=\left(\left[\begin{array}{lll}
1 & 0 & -\frac{1}{2} \\
0 & 1 & 0 \\
0 & 0 & 1
\end{array}\right], I\right), \quad \alpha=\left(\left[\begin{array}{lll}
1 & \frac{1}{2} & 0 \\
0 & 1 & 0 \\
0 & 0 & 1
\end{array}\right],\left[\begin{array}{ccc}
-1 & 0 & 0 \\
0 & 1 & 0 \\
0 & 0 & -1
\end{array}\right]\right) .
\end{aligned}
$$

Then the translation lattice is

$$
\Gamma_{2}=\Pi_{2} \cap \mathrm{Nil}=\left\langle s_{1}, s_{2}, s_{3}\right\rangle=\left\{\left[\begin{array}{lll}
1 & p & \frac{r}{2} \\
0 & 1 & q \\
0 & 0 & 1
\end{array}\right] \mid p, q, r \in Z\right\}
$$

and the holonomy group of $\Pi_{2}$ is $F_{2}=\Pi_{2} / \Gamma_{2} \cong \mathbb{Z}_{2}$, which is generated by the image $A$ of $\alpha$ under the natural map Aff(Nil) $\rightarrow$ Aut(Nil). Thus, $A$ is the automorphism on Nil defined by

$$
A=\left[\begin{array}{ccc}
-1 & 0 & 0 \\
0 & 1 & 0 \\
0 & 0 & -1
\end{array}\right]:\left[\begin{array}{lll}
1 & x & z \\
0 & 1 & y \\
0 & 0 & 1
\end{array}\right] \mapsto\left[\begin{array}{ccc}
1 & x & -z \\
0 & 1 & -y \\
0 & 0 & 1
\end{array}\right]
$$

Let $M_{2}=\Pi_{2} \backslash$ Nil be the corresponding infra-nilmanifold.

Define $\phi: \Pi_{1} \rightarrow \Pi_{2}$ by

$$
\varphi\left(\mathbf{e}_{1}\right)=\alpha \text { and } \varphi\left(\mathbf{e}_{2}\right)=\varphi\left(\mathbf{e}_{3}\right)=1_{\Pi_{2}} .
$$

Define the morphism of Lie groups $D: \mathbb{R}^{3} \rightarrow$ Nil by

$$
D(x, y, z)=\left[\begin{array}{lll}
1 & \frac{x}{2} & 0 \\
0 & 1 & 0 \\
0 & 0 & 1
\end{array}\right]
$$


Define $\mathbf{d}=1_{\mathrm{Nil}}$, then one can verify that $\phi(\gamma) \circ \lambda_{\mathbf{d}} \circ D=\lambda_{\mathbf{d}} \circ D \circ \gamma$ for $\gamma=\mathbf{e}_{1}, \mathbf{e}_{2}, \mathbf{e}_{3}$ and hence for all $\gamma \in \Pi_{1}$. Thus $\lambda_{\mathbf{d}} \circ D: \mathbb{R}^{3} \rightarrow$ Nil induces a map $f: M_{1} \rightarrow M_{2}$ so that $f_{\times}=\phi$.

Define $\psi: \Pi_{1} \rightarrow \Pi_{2}$ by

$$
\psi\left(\mathbf{e}_{1}\right)=1_{\mathrm{Nil}}, \quad \psi\left(\mathbf{e}_{2}\right)=s_{2} \text { and } \psi\left(\mathbf{e}_{3}\right)=s_{3}^{-1} .
$$

Define $\mathbf{d}^{\prime}=1_{\mathrm{Nil}} \in$ Nil and define the morphism of Lie groups $D^{\prime}: \mathbb{R}^{3} \rightarrow$ Nil by

$$
D^{\prime}(x, y, z)=\left[\begin{array}{ccc}
1 & 0 & -\frac{z}{2} \\
0 & 1 & y \\
0 & 0 & 1
\end{array}\right]
$$

Then one can verify that $\psi(\gamma) \circ \lambda_{\mathbf{d}^{\prime}} \circ D^{\prime}=\lambda_{\mathbf{d}^{\prime}} \circ D^{\prime} \circ \gamma$ for $\gamma=\mathbf{e}_{1}, \mathbf{e}_{2}, \mathbf{e}_{3}$ and hence for all $\gamma \in \Pi_{1}$. This implies that $\lambda_{\mathbf{d}^{\prime}} \circ D^{\prime}: \mathbb{R}^{3} \rightarrow$ Nil induces a map $f^{\prime}: M_{1} \rightarrow M_{2}$ so that $f_{\times}^{\prime}=\psi$.

Since $\Gamma_{1}=\Pi_{1} \cap \mathbb{R}^{3}$ is generated by $\left\{\mathbf{e}_{1}, \mathbf{e}_{2}, \mathbf{e}_{3}\right\}$ and $\Gamma_{2}=\Pi_{2} \cap$ Nil is generated by $\left\{s_{1}\right.$, $\left.s_{2}, s_{3}\right\}$, the basis $\left\{\log \left(\mathbf{e}_{1}\right), \log \left(\mathbf{e}_{2}\right), \log \left(\mathbf{e}_{3}\right)\right\}$ is a preferred basis for $\Gamma_{1}$ and $\left\{\log \left(s_{1}\right), \log \left(s_{2}\right)\right.$, $\left.\log \left(s_{3}\right)\right\}$ is a preferred basis for $\Gamma_{2}$. With respect to these preferred bases, the matrices corresponding to the induced morphisms of Lie algebras $D_{*}, D_{*}: \mathbb{R}^{3} \rightarrow \mathfrak{n i l}$ and $A_{*}: \mathfrak{n i l} \rightarrow \mathfrak{n i l}$ are

$$
D_{*}=\left[\begin{array}{ccc}
\frac{1}{2} & 0 & 0 \\
0 & 0 & 0 \\
0 & 0 & 0
\end{array}\right], \quad D_{*}^{\prime}=\left[\begin{array}{lll}
0 & 0 & 0 \\
0 & 1 & 0 \\
0 & 0 & 1
\end{array}\right], \quad A_{*}=\left[\begin{array}{ccc}
1 & 0 & 0 \\
0 & -1 & 0 \\
0 & 0 & -1
\end{array}\right] .
$$

Hence by Theorem 6.11

$$
\begin{aligned}
L\left(f, f^{\prime}\right) & =\operatorname{det}\left(D^{\prime}{ }_{*}-D_{*}\right)+\operatorname{det}\left(D^{\prime}{ }_{*}-A_{*} D_{*}\right)=-\frac{1}{2}-\frac{1}{2}=-1, \\
N\left(f, f^{\prime}\right) & =\left|\operatorname{det}\left(D^{\prime}{ }_{*}-D_{*}\right)\right|+\left|\operatorname{det}\left(D^{\prime}{ }_{*}-A_{*} D_{*}\right)\right|=1, \\
R\left(f, f^{\prime}\right) & =\sigma\left(\operatorname{det}\left(D_{*}^{\prime}{ }_{*}-D_{*}\right)\right)+\sigma\left(\operatorname{det}\left(D^{\prime}{ }_{*}-A_{*} D_{*}\right)\right)=1 .
\end{aligned}
$$

Example 7.2. In this example we will consider a 3-dimensional orientable flat Riemannian manifold $M_{1}$ and a 3-dimensional orientable infra-nilmanifold $M_{2}$.

We consider first a 3-dimensional orientable flat Riemannian manifold $M_{1}=\Pi_{1} \backslash \mathbb{R}^{3}$ where $\Pi_{1}$ is the 3 -dimensional orientable Bieberbach group $\mathfrak{G}_{2}$ [[30], Theorem 3.5.5]:

$$
\Pi_{1}=\left\langle t_{1}, t_{2}, t_{3}, \alpha \mid\left[t_{i}, t_{j}\right]=1, \quad \alpha^{2}=t_{1}, \alpha t_{2} \alpha^{-1}=t_{2}^{-1}, \alpha t_{3} \alpha^{-1}=t_{3}^{-1}\right\rangle .
$$

We can embed this group into $\operatorname{Aff}\left(\mathbb{R}^{3}\right)$ by taking $\left\{\mathbf{e}_{1}, \mathbf{e}_{2}, \mathbf{e}_{3}\right\}$ as the standard basis for $\mathbb{R}^{3}$ and

$$
t_{i}=\left(\mathbf{e}_{i}, I\right)(i=1,2,3), \quad \alpha=\left(\left[\begin{array}{c}
\frac{1}{2} \\
0 \\
0
\end{array}\right],\left[\begin{array}{ccc}
1 & 0 & 0 \\
0 & -1 & 0 \\
0 & 0 & -1
\end{array}\right]\right) .
$$

Then the translation lattice is

$$
\Gamma_{1}=\Pi_{1} \cap \mathbb{R}^{3}=\left\langle t_{1}, t_{2}, t_{3}\right\rangle=\left\langle\mathbf{e}_{1}, \mathbf{e}_{2}, \mathbf{e}_{3}\right\rangle=\mathbb{Z}^{3}
$$

and the holonomy group is $F_{1}=\Pi_{1} / \Gamma_{1} \cong \mathbb{Z}_{2}$. The holonomy group $F_{1} \subset \operatorname{Aut}\left(\mathbb{R}^{3}\right)$ is generated by $A: \mathbb{R}^{3} \rightarrow \mathbb{R}^{3}:(x, y, z) \mapsto(x,-y,-z)$. With respect to the preferred basis $\left\{\mathbf{e}_{1}, \mathbf{e}_{2}, \mathbf{e}_{3}\right\}$, the differential $A_{n}: \mathbb{R}^{3} \rightarrow \mathbb{R}^{3}$ can be expressed as a matrix as follows: 


$$
A_{*}=\left[\begin{array}{ccc}
1 & 0 & 0 \\
0 & -1 & 0 \\
0 & 0 & -1
\end{array}\right]
$$

Next we consider an infra-nilmanifold $M_{2}=\Pi_{2} \backslash$ Nil where $\Pi_{2}$ is a 3-dimensional orientable almost Bieberbach group $\pi_{5,3}$ with Seifert bundle type 5 ( [[28], Proposition 6.1], or the list of [[29], p. 800]):

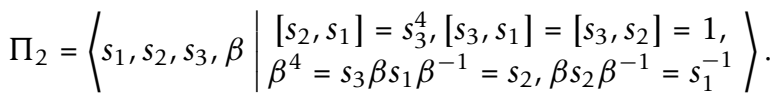

Now we can embed $\Pi_{2}$ into Aff(Nil) $=$ Nil $\rtimes$ Aut(Nil) by taking

$$
\begin{aligned}
& s_{1}=\left(\left[\begin{array}{lll}
1 & 1 & 0 \\
0 & 1 & 0 \\
0 & 0 & 1
\end{array}\right], I\right), \quad s_{2}=\left(\left[\begin{array}{lll}
1 & 0 & 0 \\
0 & 1 & 1 \\
0 & 0 & 1
\end{array}\right], I\right), \\
& s_{3}=\left(\left[\begin{array}{lll}
1 & 0 & -\frac{1}{4} \\
0 & 1 & 0 \\
0 & 0 & 1
\end{array}\right], I\right), \quad \beta=\left(\left[\begin{array}{lll}
1 & 0 & -\frac{1}{16} \\
0 & 1 & 0 \\
0 & 0 & 1
\end{array}\right],\left[\begin{array}{ccc}
1 & 0 & 0 \\
0 & 0 & -1 \\
0 & 1 & 0
\end{array}\right]\right) .
\end{aligned}
$$

Then the translation lattice is

$$
\Gamma_{2}=\Pi_{2} \cap \mathrm{Nil}=\left\langle s_{1}, s_{2}, s_{3}\right\rangle=\left\{\left[\begin{array}{lll}
1 & p & \frac{r}{4} \\
0 & 1 & q \\
0 & 0 & 1
\end{array}\right] \mid p, q, r \in Z\right\}
$$

and the holonomy group of $\Pi_{2}$ is $F_{2}=\Pi_{2} / \Gamma_{2} \cong \mathbb{Z}_{4}$. The holonomy group $F_{2} \subset$ Aut (Nil) is generated by the image $B$ of $\beta$ under the natural map Aff(Nil) $\rightarrow$ Aut(Nil). Thus, $B$ is the automorphism on Nil defined by

$$
B=\left[\begin{array}{ccc}
1 & 0 & 0 \\
0 & 0 & -1 \\
0 & 1 & 0
\end{array}\right]:\left[\begin{array}{lll}
1 & x & z \\
0 & 1 & y \\
0 & 0 & 1
\end{array}\right] \mapsto\left[\begin{array}{ccc}
1 & -y & -x z \\
0 & 1 & x \\
0 & 0 & 1
\end{array}\right] .
$$

Hence the differential $B$ * of $B$ is given by

$$
B_{*}:\left[\begin{array}{lll}
0 & u & w \\
0 & 0 & v \\
0 & 0 & 0
\end{array}\right] \mapsto\left[\begin{array}{ccc}
0 & -v & w \\
0 & 0 & u \\
0 & 0 & 0
\end{array}\right] .
$$

With respect to the preferred basis $\left\{\log s_{1}, \log s_{2}, \log s_{3}\right\}$ of nil, the differential $B_{*}$ can be written as a matrix as follows:

$$
B_{*}=\left[\begin{array}{ccc}
0 & -1 & 0 \\
1 & 0 & 0 \\
0 & 0 & 1
\end{array}\right] \text {. }
$$

Now define a morphism of groups $\phi: \Pi_{1} \rightarrow \Pi_{2}$ by

$$
\varphi\left(t_{1}\right)=s_{3}, \varphi\left(t_{2}\right)=s_{2}, \varphi\left(t_{3}\right)=1_{\mathrm{Nil}}, \varphi(\alpha)=\beta^{2} .
$$

Also define $\mathbf{d}=\mathbf{1}_{\mathrm{Nil}}$ and define the morphism of Lie groups $D$ by

$$
D: \mathbb{R}^{3} \rightarrow \mathrm{Nil}:(x, y, z) \mapsto\left[\begin{array}{ccc}
1 & 0 & -x / 4 \\
0 & 1 & y \\
0 & 0 & 1
\end{array}\right] .
$$


Then $\phi(\gamma) \circ \lambda_{\mathbf{d}} \circ D=\lambda_{\mathbf{d}} \circ D \circ \gamma$ for $\gamma=t_{1}, t_{2}, t_{3}, \alpha$. Hence this equation holds for every $\gamma \in \Pi_{1}$ and $\lambda_{\mathbf{d}} \circ D: \mathbb{R}^{3} \rightarrow$ Nil induces a continuous map $f: M_{1} \rightarrow M_{2}$ so that $f_{\times}$ $=\phi$. With respect to the preferred bases chosen above, the differential $D$ * can be written as a matrix as follows:

$$
D_{*}=\left[\begin{array}{lll}
0 & 0 & 0 \\
0 & 1 & 0 \\
1 & 0 & 0
\end{array}\right] .
$$

Similarly, by defining the morphism of groups $\psi: \Pi_{1} \rightarrow \Pi_{2}$ by

$$
\psi\left(t_{1}\right)=s_{3}^{-1}, \quad \psi\left(t_{2}\right)=1, \quad \psi\left(t_{3}\right)=s_{1}, \quad \psi(\alpha)=\beta^{-2},
$$

one can show that $\lambda_{\mathbf{d}^{\prime}} \circ D^{\prime}: \mathbb{R}^{3} \rightarrow$ Nil induces a continuous map $f^{\prime}: M_{1} \rightarrow M_{2}$ so that $f_{\times}^{\prime}=\psi$, where

$$
\begin{gathered}
\mathbf{d}^{\prime}=1_{\mathrm{Nil}}, \\
D^{\prime}=\mathbb{R}^{3} \rightarrow \mathrm{Nil}:(x, y, z) \mapsto\left[\begin{array}{ccc}
1 & z & x / 4 \\
0 & 1 & 0 \\
0 & 0 & 1
\end{array}\right] .
\end{gathered}
$$

With respect to the same preferred bases, $D{ }_{*}$ can be written as a matrix as follows:

$$
D^{\prime}{ }_{*}=\left[\begin{array}{ccc}
0 & 0 & 1 \\
0 & 0 & 0 \\
-1 & 0 & 0
\end{array}\right] .
$$

Hence $\operatorname{det}\left(D^{\prime}{ }_{*}-D_{*}\right)=-2, \operatorname{det}\left(D^{\prime}{ }_{*}-B_{*} D_{*}\right)=0, \operatorname{det}\left(D^{\prime}{ }_{*}-B_{*}^{2} D_{*}\right)=2$ and $\operatorname{det}\left(D^{\prime}{ }_{*}-B_{*}^{3} D_{*}\right)=0$. From the formulas in Theorem 6.11, it follows that

$$
L\left(f, f^{\prime}\right)=0, \quad N\left(f, f^{\prime}\right)=2, \quad R\left(f, f^{\prime}\right)=\infty .
$$

\section{Acknowledgements}

The authors would like to thank the referee for pointing out some errors and making careful corrections to a few expressions in the original version of the article. The second-named author is partially supported by the Mid-career Researcher Program through NRF grant funded by the MEST (No. 2010-0008640) and by the Sogang University Research Grant of 2010. The third-named author is supported by a Ph.D. fellowship of the Research FoundationFlanders (FWO)

\section{Author details}

${ }^{1}$ Department of Mathematics, Sogang University, Seoul 121-742, Korea ${ }^{2}$ Department of Mathematics, Katholieke Universiteit Leuven, Campus Kortrijk, B-8500 Kortrijk, Belgium

\section{Authors' contributions}

All authors contributed equally and significantly in writing this paper. All authors read and approved the final manuscript.

\section{Competing interests}

The authors declare that they have no competing interests.

Received: 3 December 2011 Accepted: 14 March 2012 Published: 14 March 2012

\section{References}

1. Wecken, F: Fixpunktklassen. III. Mindestzahlen von Fixpunkten. Math Ann. 118, 544-577 (1942)

2. Hart, EL: Algebraic techniques for calculating the Nielsen number on hyperbolic surfaces. Handbook of Topological Fixed Point Theory. pp. 463-487.Springer, Dordrecht (2005)

3. McCord, CK: Computing Nielsen numbers, (English summary) Nielsen theory and dynamical systems (South Hadley, MA, 1992). Contemp Math Am Math Soc Providence RI. 152, 249-267 (1993)

4. Wong, P: Fixed Point Theory for Homogeneous Spaces-A Brief Survey. Handbook of Topological Fixed Point Theory. pp. 265-283.Springer, Dordrecht (2005) 
5. Anosov, DV: The Nielsen numbers of maps on nil-manifolds. Uspekhi Mat Nauk 40, 133-134 (1985). (in Russian); English translation: Russ. Math. Surv. 40, 149-150 (1985)

6. Fadell, E, Husseini, S: On a Theorem of Anosov on Nielsen Numbers for Nilmanifolds, Nonlinear Functional Analysis and its Applications (Maratea, 1985). In NATO Adv Sci Inst Ser C Math Phys Sci, vol. 173, pp. 47-53.Reidel, Dordrecht (1986)

7. Dekimpe, K, Penninckx, P: Coincidence theory for infra-nilmanifolds. Topol Appl. 157, 1815-1832 (2010). doi:10.1016/j. topol.2010.02.019

8. Lee, KB: Maps on infra-nilmanifolds. Pac J Math. 168, 157-166 (1995)

9. McCord, CK: Lefschetz and Nielsen coincidence numbers on nilmanifolds and solvmanifolds, II. Topol Appl. 75, 81-92 (1997). doi:10.1016/S0166-8641(96)00081-8

10. McCord, CK: Estimating Nielsen numbers on infrasolvmanifolds. Pac J Math. 154, 345-368 (1992)

11. Kim, SW, Lee, JB, Lee, KB: Averaging formula for Nielsen numbers. Nagoya Math J. 178, 37-53 (2005)

12. Lee, JB, Lee, KB: Lefschetz numbers for continuous maps, and periods for expanding maps on infra-nilmanifolds. J Geom Phys. 56, 2011-2023 (2006). doi:10.1016/j.geomphys.2005.11.003

13. Ha, KY, Lee, JB, PenninckX, P: Anosov theorem for coincidences on special solvmanifolds of type (R). P Am Math Soc. 139, 2239-2248 (2011). doi:10.1090/S0002-9939-2010-10721-9

14. Kim, SW, Lee, JB: Averaging formula for Nielsen coincidence numbers. Nagoya Math J. 186, 69-93 (2007)

15. Gonçalves, DL: Coincidence theory. Handbook of Topological Fixed Point Theory. pp. 3-42.Springer, Dordrecht (2005)

16. Schirmer, H: Mindestzahlen von Koinzidenzpunkten. J Reine Angew Math. 194, $21-39$ (1955)

17. Staecker, PC: On the uniqueness of the coincidence index on orientable differentiable manifolds. Topol Appl. 154, 1961-1970 (2007). doi:10.1016/j.topol.2007.02.003

18. Dekimpe, K: Almost-Bieberbach Groups: Affine and Polynomial Structures. In Lect Notes in Math, vol. 1639,SpringerVerlag, Berlin (1996)

19. Lee, KB, Raymond, F: Rigidity of almost crystallographic groups. Contemp Math Am Math Soc. 44, 73-78 (1985)

20. Wong, P: Reidemeister number, Hirsch rank, coincidences on polycyclic groups and solvmanifolds. J reine angew Math. 524, 185-204 (2000)

21. Dekimpe, $K$, Penninckx, P: The finiteness of the Reidemeister number of morphisms between almost-crystallographic groups. J Fixed Point Theory Appl. 9, 257-283 (2011). doi:10.1007/s11784-011-0043-2

22. Jiang, B: Lectures on Nielsen fixed point theory. Contemp Math Am Math Soc. 14 (1983)

23. Jezierski, J: The Nielsen number product formula for coincidences. Fund Math. 134, 183-212 (1990)

24. Kim, SW, Lee, JB: Anosov theorem for coincidences on nilmanifolds. Fund Math. 185, 247-259 (2005). doi:10.4064/ fm185-3-3

25. Raghunathan, MS: Discrete subgroups of Lie groups, Ergebnisse der Mathematik und ihrer Grenzgebiete. SpringerVerlag, New York68 (1972)

26. Gonçalves, DL, Wong, PN-S: Nilmanifolds are Jiang-type spaces for coincidences. Forum Math. 13(1):133-141 (2001)

27. Ha, KY, Lee, JB: Left invariant metrics and curvatures on simply connected three-dimensional Lie groups. Math Nachr. 282, 868-898 (2009). doi:10.1002/mana.200610777

28. Dekimpe, K, Igodt, P, Kim, S, Lee, KB: Affine structures for closed 3-dimensional manifolds with Nil-geometry. Quart J Math Oxford. 46(2):141-167 (1995). doi:10.1093/qmath/46.2.141

29. Choi, D, Shin, J: Free actions of finite Abelian groups on 3-dimensional nilmanifolds. J Korean Math Soc. 42, 795-826 (2005)

30. Wolf, J: Spaces of Constant Curvature. Publish or Perish, Wilmington, 5 (1984)

doi:10.1186/1687-1812-2012-39

Cite this article as: $\mathrm{Ha}$ et al:: Formulas for the Reidemeister, Lefschetz and Nielsen coincidence number of maps between infra-nilmanifolds. Fixed Point Theory and Applications 2012 2012:39.

\section{Submit your manuscript to a SpringerOpen ${ }^{\circ}$ journal and benefit from:}

- Convenient online submission

- Rigorous peer review

- Immediate publication on acceptance

- Open access: articles freely available online

- High visibility within the field

- Retaining the copyright to your article

Submit your next manuscript at $\gg$ springeropen.com 